\title{
Iterative stability analysis of spatial domain decomposition based on block Jacobi algorithm for the diamond-difference scheme
}

\author{
Dmitriy Y. Anistratov*, Yousry Y. Azmy \\ Department of Nuclear Engineering, North Carolina State University Raleigh, NC 27695-7909, United States
}

\section{A R T I C L E I N F O}

\section{Article history:}

Received 17 August 2014

Received in revised form 7 April 2015

Accepted 18 May 2015

Available online 28 May 2015

\section{Keywords:}

Particle transport equation

Radiative transfer equation

Domain decomposition

Iterative methods

Fourier analysis

\begin{abstract}
A B S T R A C T
We study convergence of the integral transport matrix method (ITMM) based on a parallel block Jacobi (PBJ) iterative strategy for solving particle transport problems. The ITMM is a spatial domain decomposition method proposed for massively parallel computations. A Fourier analysis of the PBJ-based iterations applied to $S_{N}$ diamond-difference equations in 1D slab and 2D Cartesian geometries is performed. It is carried out for infinite-medium problems with homogeneous material properties. To analyze the performance of the ITMM with the PBJ algorithm and evaluate its potential in scalability we consider a limiting case of one spatial cell per subdomain. The analysis shows that in such limit the spectral radius of the iteration method is one without regard to values of the scattering ratio and optical thickness of the spatial cells. This implies lack of convergence in infinite medium. Numerical results of finite-medium problems are presented. They demonstrate effects of finite size of spatial domain on the performance of the iteration algorithm as well as its asymptotic behavior when the extent of the spatial domain increases. These numerical experiments also show that for finite domains iterative convergence to a finite criterion is achievable in a multiple of the sum of number of cells in each dimension.
\end{abstract}

(c) 2015 Elsevier Inc. All rights reserved.

\section{Introduction}

A wide variety of physical problems involves transport of particles in matter when they interact with a background medium but collisions between particles themselves has a negligible effect on the transport process. The mathematical description of such kind of particle transport phenomena is based on the linear Boltzmann equation. It is referred to as particle transport or radiative transfer equation depending on the underlying physical processes. These problems have a lot of applications in science and engineering [1,2]. The transport equation is a foundation for mathematical models of radiative transfer in plasmas and atmospheres, neutron transport in nuclear reactors and well-logging problems, photon and electron transport in cancer therapy problems.

The transport equation is a formulation of detailed particle balance in phase space. If particles are treated classically, then their distribution depends on spatial position $(\mathbf{r})$, direction of motion $(\boldsymbol{\Omega})$, energy $(E)$, and time $(t)$. The solution of the transport equation of various forms is related to the particle distribution function $n(\mathbf{r}, \boldsymbol{\Omega}, E, t)$. For example, the neutron

\footnotetext{
* Corresponding author.

E-mail addresses: anistratov@ncsu.edu (D.Y. Anistratov), yyazmy@ncsu.edu (Y.Y. Azmy).
} 
transport equation is formulated for the particle angular flux $\psi=v n$, where $v$ is the particle speed. The radiative transfer equation has a form similar to one for neutrons and defined for the specific intensity $I=\operatorname{chvn}$, where $v$ is the photon frequency and $c$ is the speed of light. When one simulates processes in a physical system, the transport equation is just a part of a large system of nonlinearly coupled differential equations describing multiphysical phenomena. It enables one to model processes associated with dynamic energy redistribution in the system governed by particle transport and interaction with matter.

The steady-state one-group particle transport equation has the following form:

$$
\begin{aligned}
& \mathcal{L} \psi(\mathbf{r}, \boldsymbol{\Omega})=\mathcal{S} \psi(\mathbf{r}, \boldsymbol{\Omega})+\frac{1}{4 \pi} q(\mathbf{r}), \quad \mathbf{r} \in \mathbf{D}, \quad \text { for all } \boldsymbol{\Omega}, \\
& \left.\psi\right|_{\mathbf{r} \in \partial \mathbf{D}}=\psi^{i n}\left(\mathbf{r}_{\gamma}, \boldsymbol{\Omega}\right), \quad \text { for } \mathbf{r}_{\gamma} \in \partial \mathbf{D} \text { and } \boldsymbol{\Omega} \cdot \mathbf{e}_{n}<0,
\end{aligned}
$$

where $\mathbf{D}$ is the spatial domain, $\mathbf{e}_{n}$ is outward normal at the external boundary $\partial \mathbf{D}$. The operator $\mathcal{L}$ accounts for particle streaming and collisions

$$
\mathcal{L} \psi \equiv \boldsymbol{\Omega} \cdot \nabla \psi(\mathbf{r}, \boldsymbol{\Omega})+\sigma_{t}(\mathbf{r}) \psi(\mathbf{r}, \boldsymbol{\Omega}),
$$

where $\sigma_{t}$ is the total cross sections. The integral operator $\mathcal{S}$ represents scattering processes and in case of isotropic scattering is given by

$$
\mathcal{S} \psi \equiv \frac{1}{4 \pi} \sigma_{S}(\mathbf{r}) \int_{4 \pi} \psi(\mathbf{r}, \boldsymbol{\Omega}) d \boldsymbol{\Omega}=\frac{1}{4 \pi} \sigma_{S}(\mathbf{r}) \phi(\mathbf{r}),
$$

where $\sigma_{s}$ is the scattering cross section and

$$
\phi(\mathbf{r})=\int_{4 \pi} \psi(\mathbf{r}, \boldsymbol{\Omega}) d \boldsymbol{\Omega}
$$

is scalar flux. $q$ is an isotropic source of particles. Except for highly idealized models, the transport equation can be solved only by means of numerical methods. The essential feature of the transport equation is that the phase space is of high dimensionality. In many practical applications discretization of the transport equation leads to a very large number of unknowns in the phase space. Computational transport problems with more than $10^{10}$ unknowns are rather common nowadays. To solve this type of numerical problems one relies on massively parallel computations using high-performance computers. Parallel methodologies for transport problems are based on various transport sweeping algorithms and domain decomposition methods [3-10].

Let us consider that the spatial domain $\mathbf{D}$ of the transport problem (1) is divided into a number of subdomains $\mathbf{D}_{l}$ $\left(\mathbf{D}=\bigcup_{l} \mathbf{D}_{l}\right)$. We apply spatial domain decomposition to the transport equation in a discretized form

$$
\begin{aligned}
& \mathcal{L}_{h} \boldsymbol{\psi}_{h, l}=\mathcal{S}_{h} \boldsymbol{\psi}_{h, l}+\frac{1}{4 \pi} q_{h}, \quad \mathbf{r}_{h} \in \mathbf{D}_{l}, \quad \text { for all } \boldsymbol{\Omega}_{m}, \\
& \left.\boldsymbol{\psi}_{h, l}\right|_{\mathbf{r}_{h} \in \partial \mathbf{D}_{l}}=\left.\boldsymbol{\psi}_{h, l^{*}}\right|_{\mathbf{r}_{h} \in \partial \mathbf{D}_{l}}, \quad \text { for } \boldsymbol{\Omega}_{m} \cdot \mathbf{e}_{n, l}<0, \\
& l=1, \ldots, N_{l},
\end{aligned}
$$

where $\mathcal{L}_{h}$ and $\mathcal{S}_{h}$ are discretized operators $\mathcal{L}$ and $\mathcal{S}$, respectively, $\psi_{h, l}$ is the vector of discrete angular flux in subdomain $\mathbf{D}_{l}, \mathbf{r}_{h}$ defines the given spatial grid, $\boldsymbol{\Omega}_{m}$ is a discrete-ordinate direction, $\mathbf{e}_{n, l}$ is outward normal at the boundary of $l$-th subdomain, $l^{*}$ is the index of neighboring subdomain. The transport problem (6) can be solved locally in each subdomain with a prescribed lagged angular fluxes coming from surrounding subdomains. The integral transport matrix method (ITMM) is a spatial domain decomposition method for solving the discretized particle transport equation [11-14]. It uses an integral form of Eqs. (6) and applies response-matrix approach [15,16] to formulate of the equations for the scalar flux in $l$-th subdomain

$$
\boldsymbol{\phi}_{h, l}=\mathcal{J}_{\phi, h} \boldsymbol{\phi}_{h, l}+\mathcal{B}_{\phi, h} q_{h}+\mathcal{K}_{\phi, h} \boldsymbol{\psi}_{h, l}^{i n}, \quad \text { for } \quad \mathbf{r}_{h} \in \mathbf{D}_{l},
$$

and for the outgoing angular flux at the subdomain boundary

$$
\boldsymbol{\psi}_{h, l}^{\text {out }}=\mathcal{J}_{\psi, h} \boldsymbol{\phi}_{h, l}+\mathcal{B}_{\psi, h} q_{h}+\mathcal{K}_{\psi, h} \boldsymbol{\psi}_{h, l}^{\text {in }} \text { for } \quad \mathbf{r}_{h} \in \partial \mathbf{D}_{l},
$$

where

$$
\begin{aligned}
& \boldsymbol{\psi}_{h, l}^{\text {out }}=\left.\boldsymbol{\psi}_{h, l}\right|_{\mathbf{r}_{h} \in \partial \mathbf{D}_{l}} \text { for } \quad \boldsymbol{\Omega}_{m} \cdot \mathbf{e}_{n, l}>0, \\
& \boldsymbol{\psi}_{h, l}^{\text {in }}=\boldsymbol{\psi}_{h, l^{*}}^{\text {out }}=\left.\boldsymbol{\psi}_{h, l^{*}}\right|_{\mathbf{r}_{h} \in \partial \mathbf{D}_{l}} \text { for } \quad \boldsymbol{\Omega}_{m} \cdot \mathbf{e}_{n, l}<0,
\end{aligned}
$$

$\mathcal{J}_{\alpha, h}, \mathcal{B}_{\alpha, h}$, and $\mathcal{K}_{\alpha, h}(\alpha=\psi, \phi)$ are corresponding discrete operators. The ITMM uses parallel block Jacobi (PBJ) algorithm to solve the transport problem in $\mathbf{D}$ that is defined as follows: 


$$
\begin{aligned}
& \left(\mathcal{I}-\mathcal{J}_{\phi, h}\right) \boldsymbol{\phi}_{h, l}^{(s)}=\mathcal{B}_{\phi, h} q_{h}+\mathcal{K}_{\phi, h} \boldsymbol{\psi}_{h, l}^{\text {in }(s-1)}, \quad \text { for } \quad \mathbf{r}_{h} \in \mathbf{D}_{l}, \\
& \boldsymbol{\psi}_{h, l}^{\text {out }(s)}=\mathcal{J}_{\psi, h} \boldsymbol{\phi}_{h, l}^{(s)}+\mathcal{B}_{\psi, h} q_{h}+\mathcal{K}_{\psi, h} \boldsymbol{\psi}_{h, l}^{\text {in }(s-1)} \text { for } \quad \mathbf{r}_{h} \in \partial \mathbf{D}_{l}, \\
& l=1, \ldots, N_{l},
\end{aligned}
$$

where $s$ is the iterations index. We note that this iterative algorithm (8) is equivalent to the PBJ iterative procedure applied to the set of local problems (6) in subdomains in the following form:

$$
\begin{aligned}
& \boldsymbol{\psi}_{h, l}^{(s)}=\mathcal{L}_{h}^{-1}\left(\hat{\mathcal{S}}_{h} \boldsymbol{\phi}_{h, l}^{(s)}+\frac{1}{4 \pi} q_{h}\right), \quad \mathbf{r}_{h} \in \mathbf{D}_{l}, \\
& \left.\boldsymbol{\psi}_{h, l}^{(s)}\right|_{\mathbf{r}_{h} \in \partial \mathbf{D}_{l}}=\left.\boldsymbol{\psi}_{h, l^{*}}^{(s-1)}\right|_{\mathbf{r}_{h} \in \partial \mathbf{D}_{l}}, \quad \text { for } \boldsymbol{\Omega}_{m} \cdot \mathbf{e}_{n, l}<0, \\
& \boldsymbol{\phi}_{h, l}^{(s)}=\mathcal{M}_{h} \boldsymbol{\psi}_{h, l}^{(s)}, \\
& \hat{\mathcal{S}}_{h}=\mathcal{S}_{h} \mathcal{M}_{h}^{-1} .
\end{aligned}
$$

This formulation can be used to analyze the PBJ algorithm.

There are two main components of computational costs associated with using the ITMM with the PBJ algorithm for parallel calculations: (i) computational efforts in solving a local transport problem in a subdomain that is assigned to a single processor, (ii) number of PBJ iterations for solving the global transport problem. The smaller a subdomain the faster a local problem can be solved because it is related to the number of unknowns in the subdomain. To analyze potential of this methodology in scaling it is necessary to study convergence rate of the PBJ method. The asymptotic case from the viewpoint of scaling is a subdomain that consists of a single cell of spatial grid. Thus analysis of such a limiting case will enable one to study the performance of the ITMM with the PBJ algorithm in parallel computations.

Recently the ITMM with the PBJ algorithm was applied for solving the transport equation approximated by the diamonddifference (DD) scheme [13]. The studies of strong scaling has been performed for this case. In such analysis, one considers a transport problem with a given spatial domain that is solved with increasing number of processors each of which is assigned a single subdomain. The results showed that the number of PBJ iterations increases with increase in number subdomains while the computational cost per iteration decreases. It was also observed that there exits some optimum number of processors and hence subdomains that results in a minimum execution time in a given problem.

In this paper we perform Fourier analysis of the ITMM with the PBJ algorithm applied to the DD scheme considering the limiting case of one spatial cell per subdomain. Note that results of Fourier analysis of the DD equations solved with different iterative methods are presented in $[17,18]$. We consider one-group transport problems with isotropic scattering and theoretically analyze the domain decomposition algorithm in 1D slab and 2D Cartesian geometries. The Fourier analysis of homogeneous infinite medium $S_{N}$ problems enables us to determine the spectral radius of the iterative process. A detailed analysis is carried out for the $S_{2}$ case that provides a set of analytical results. We study properties of eigenvalues and associated eigenvectors in 2D. Numerical results of finite-medium problems are presented to show effects of finite size of the spatial domain on the iterative properties of the ITMM. They also demonstrate its asymptotic behavior when the spatial domain increases in size and leakage at the global boundaries diminishes.

The remainder of this paper is organized as follows. In Section 2 we formulate the ITMM with the PBJ algorithm for the DD scheme in 1D slab geometry and perform Fourier analysis for an infinite homogeneous medium. In Section 3 we describe the iterative algorithm in 2D Cartesian geometry and apply Fourier analysis for the resulting iteration equations. We analyze $S_{2}$ problems to get analytic results in Section 4.1. The results of the Fourier analysis of $S_{N}$ case are presented in Section 4.2. Numerical results for finite-medium problems are presented in Section 5. We conclude with a discussion in Section 6.

\section{Analysis in 1D slab geometry}

\subsection{Formulation of the method}

The steady-state one-group transport equation in 1D slab geometry in case of isotropic scattering and external source is given by

$$
\begin{aligned}
& \mu \frac{\partial \psi(x, \mu)}{\partial x}+\sigma_{t}(x) \psi(x, \mu)=\frac{1}{2}\left(\sigma_{s}(x) \phi(x)+q(x)\right), \quad x \in \mathcal{D}, \\
& \mathcal{D}=\{0<x<X\}, \quad-1 \leq \mu \leq 1, \\
& \left.\psi\right|_{x=0}=\psi_{i n, L}(\mu) \quad \text { for } \quad \mu>0,\left.\quad \psi\right|_{x=X}=\psi_{i n, R}(\mu) \text { for } \mu<0,
\end{aligned}
$$

where

$$
\phi(x)=\int_{-1}^{1} \psi(x, \mu) d \mu,
$$


$x$ is the spatial position, $\mu$ is the directional cosine, $\psi_{i n, L}$ and $\psi_{i n, R}$ are angular fluxes of incoming particles. The DD method for discretization of Eq. (10) is defined as follows [15]:

$$
\begin{aligned}
& \frac{\mu_{m}}{\Delta x_{k}}\left(\psi_{m, k}^{+}-\psi_{m, k}^{-}\right)+\sigma_{t, k} \psi_{m, k}=\frac{1}{2}\left(\sigma_{s, k} \phi_{k}+q_{k}\right), \\
& \psi_{m, k}=\frac{1}{2}\left(\psi_{m, k}^{+}+\psi_{m, k}^{-}\right), \\
& \phi_{k}=\sum_{m=1}^{M} \psi_{m, k} w_{m}, \\
& \psi_{m, k}^{-}=\psi_{m, k \mp 1 / 2} \text { for } \mu_{m} \gtrless 0, \\
& \psi_{m, k}^{+}=\psi_{m, k \pm 1 / 2} \text { for } \mu_{m} \gtrless 0 .
\end{aligned}
$$

Here $m$ is the index of discrete angular direction, $k$ is the index of spatial cells, $\psi_{m, k}$ is the $m$-th angular flux averaged over the $k$-th spatial cell, $\psi_{m, k \pm 1 / 2}$ are the cell-edge angular fluxes, $\sigma_{t, k}, \sigma_{s, k}, q_{k}$ are cell-average values of the total and scattering cross sections and the source, respectively, $w_{m}$ is the $m$-th angular quadrature weight, $\Delta x_{k}$ is width the $k$-th cell.

In our analysis, we consider domain decomposition with one spatial cell per subdomain. The ITMM formulation starts a new iteration by importing the incoming angular fluxes $\left(\psi_{m, k}^{-}\right)$from the previous iteration, but unlike source iterations it fully resolves the scattering source. Hence, the ITMM with the PBJ algorithm applied to Eqs. (12) yields the iteration scheme given by

$$
\begin{aligned}
& \frac{\mu_{m}}{\Delta x}\left(\psi_{m, k}^{+(s)}-\psi_{m, k}^{-(s-1)}\right)+\sigma_{t, k} \psi_{m, k}^{(s)}=\frac{1}{2}\left(\sigma_{s, k} \phi_{k}^{(s)}+q_{k}\right), \\
& \psi_{m, k}^{(s)}=\frac{1}{2}\left(\psi_{m, k}^{+(s)}+\psi_{m, k}^{-(s-1)}\right), \\
& \phi_{k}^{(s)}=\sum_{m=1}^{M} \psi_{m, k}^{(s)} w_{m} .
\end{aligned}
$$

We define the errors in the $s$-th iterate

$$
\delta \boldsymbol{\psi}^{(s)}=\boldsymbol{\psi}-\boldsymbol{\psi}^{(s)}, \quad \delta \boldsymbol{\phi}^{(s)}=\boldsymbol{\phi}-\boldsymbol{\phi}^{(s)}
$$

of the vector of discrete transport solution to the DD equations (12). Subtracting Eqs. (14) from the corresponding Eqs. (12) and using (15) we obtain the equations for the error in the $s$-th iterate of ITMM. In a homogeneous medium these equations are given by

$$
\begin{aligned}
& \hat{v}_{m, k}\left(\delta \psi_{m, k}^{+(s)}-\delta \psi_{m, k}^{-(s-1)}\right)+\delta \psi_{m, k}^{(s)}=\frac{1}{2} c_{k} \delta \phi_{k}^{(s)}, \\
& \delta \psi_{m, k}^{(s)}=\frac{1}{2}\left(\delta \psi_{m, k}^{+(s)}+\delta \psi_{m, k}^{-(s-1)}\right), \\
& \delta \phi_{k}^{(s)}=\sum_{m=1}^{M} \delta \psi_{m}^{(s)} w_{m}, \\
& \delta \psi_{m, k}^{-(s-1)}=\psi_{m, k \mp 1 / 2}^{(s-1)} \text { for } \mu_{m} \gtrless 0 \\
& \psi_{m, k}^{+(s)}=\psi_{m, k \pm 1 / 2}^{+(s)} \text { for } \mu_{m} \gtrless 0
\end{aligned}
$$

where

$$
\hat{v}_{m, k}=\frac{\mu_{m}}{\sigma_{t, k} \Delta x_{k}}, \quad c_{k}=\frac{\sigma_{s, k}}{\sigma_{t, k}} .
$$

Here $c_{k}$ is the scattering ratio in the $k$-th cell. We use Eq. (16b) to eliminate the cell-averaged quantities in Eq. (16a) to obtain the equations for the iterative errors only in cell-edge angular fluxes:

$$
\begin{aligned}
& \left(1+2 \hat{v}_{m, k}-\frac{1}{2} c_{k} w_{m}\right) \delta \psi_{m, k}^{+(s)}-\frac{c_{k}}{2} \sum_{m^{\prime} \neq m} \delta \psi_{m^{\prime}, k}^{+(s)} w_{m^{\prime}} \\
& =\frac{c_{k}}{2} \sum_{m^{\prime} \neq m} \delta \psi_{m^{\prime}, k}^{-(s-1)} w_{m^{\prime}}-\left(1-2 \hat{v}_{m, k}-\frac{1}{2} c_{k} w_{m}\right) \delta \psi_{m, k}^{-(s-1)}
\end{aligned}
$$




\subsection{Fourier analysis}

To analyze the performance of the ITMM we consider problems with infinite homogeneous medium and uniform spatial grid and hence $\Delta x_{k}=\Delta x, c_{k}=c, \hat{v}_{m, k}=\hat{v}_{m}$. We introduce a Fourier ansatz:

$$
\delta \psi_{m, k+1 / 2}^{(s)}=\omega^{s}(\lambda) v_{m}(\lambda) e^{i \sigma_{t} \lambda x_{k+1 / 2}}
$$

considering a single Fourier error mode with an arbitrary $\lambda$. We substitute (20) in Eq. (19) and take into account Eqs. (17). As a result, we obtain the equations for $\omega$ and the vector of coefficients of the Fourier expansion (20)

$$
\mathbf{v}=\left(v_{1}, \ldots, v_{M}\right)^{T}
$$

of the following matrix form:

$$
\omega \mathbf{G}^{+} \mathbf{v}=\mathbf{G}^{-} \mathbf{v},
$$

where

$$
\mathbf{G}^{ \pm}= \pm \mathbf{C}^{ \pm} \mathbf{F}^{ \pm}
$$

and

$$
\begin{aligned}
& \mathbf{C}^{ \pm}=\left(\begin{array}{ccccccc}
\hat{p}_{1}^{ \pm} & -\hat{c}_{2} & \ldots & & & & -\hat{c}_{M} \\
-\hat{c}_{1} & \hat{p}_{2}^{ \pm} & -\hat{c}_{3} & \ldots & & & -\hat{c}_{M} \\
\vdots & & \ddots & & & & \vdots \\
-\hat{c}_{1} & \ldots & -\hat{c}_{m-1} & \hat{p}_{m}^{ \pm} & -\hat{c}_{m+1} & \ldots & -\hat{c}_{M} \\
\vdots & & & & \ddots & & \vdots \\
-\hat{c}_{1} & \ldots & & & -\hat{c}_{M-2} & \hat{p}_{M-1}^{ \pm} & -\hat{c}_{M} \\
-\hat{c}_{1} & \ldots & & & & -\hat{c}_{M-1} & \hat{p}_{M}^{ \pm}
\end{array}\right), \\
& \mathbf{F}^{ \pm}=\operatorname{diag}\left(e^{ \pm i \hat{\tau}_{1}}, \ldots, e^{ \pm i \hat{\tau}_{M}}\right), \\
& \hat{p}_{m}^{ \pm}=1 \pm 2 \hat{v}_{m}-\hat{c}_{m}, \quad \hat{c}_{m}=\frac{1}{2} c w_{m}, \\
& \hat{\tau}_{m}=\frac{1}{2} \hat{\varkappa}_{m} \tilde{\lambda}, \\
& \tilde{\lambda}=\lambda \sigma_{t} \Delta x, \\
& \hat{\varkappa}_{m}= \begin{cases}1 & \text { for } \mu_{m}>0, \\
-1 & \text { for } \mu_{m}<0 .\end{cases}
\end{aligned}
$$

Thus in a general $S_{N}$ case $\omega$ is the eigenvalue of the matrix

$$
\left(\mathbf{G}^{+}\right)^{-1} \mathbf{G}^{-} \text {. }
$$

The spectral radius is given by

$$
\rho=\sup _{\lambda}|\omega(\lambda)| \text {. }
$$

We now consider the $S_{2}$ case to get analytic results. In $S_{2}$ problems there are two directions $(M=2)$ and

$$
w_{m}=1, \quad\left|\mu_{m}\right|=\frac{1}{\sqrt{3}}, \quad m=1,2 .
$$

The equation for the eigenvalue $\omega$ can be cast as

$$
\xi^{+}\left(\xi^{+}-c\right) \omega^{2}+2 \cos \tilde{\lambda}\left(\xi^{+} \xi^{-}-c\right) \omega+\xi^{-}\left(\xi^{-}-c\right)=0,
$$

where

$$
\xi^{ \pm}=1 \pm \frac{2}{\sqrt{3} \sigma_{t} \Delta x} .
$$

Considering the limit of Eq. (33) for infinitely optically thin and thick spatial cells we get that

$$
\begin{aligned}
& \omega=e^{ \pm i \tilde{\lambda}} \quad \text { as } \quad \sigma_{t} \Delta x \rightarrow 0, \\
& \omega=-e^{ \pm i \tilde{\lambda}} \quad \text { as } \quad \sigma_{t} \Delta x \rightarrow \infty,
\end{aligned}
$$




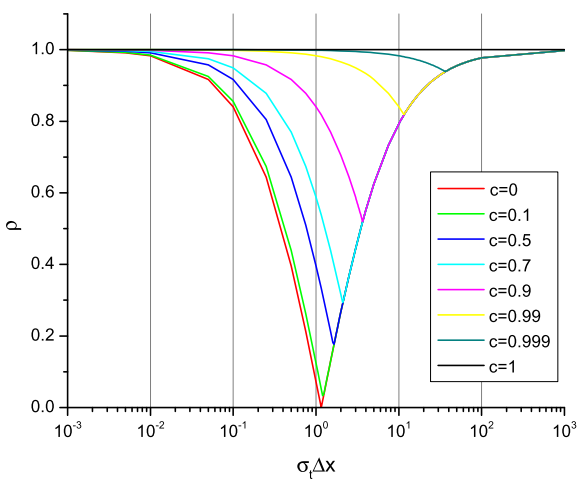

(a) $S_{2}$

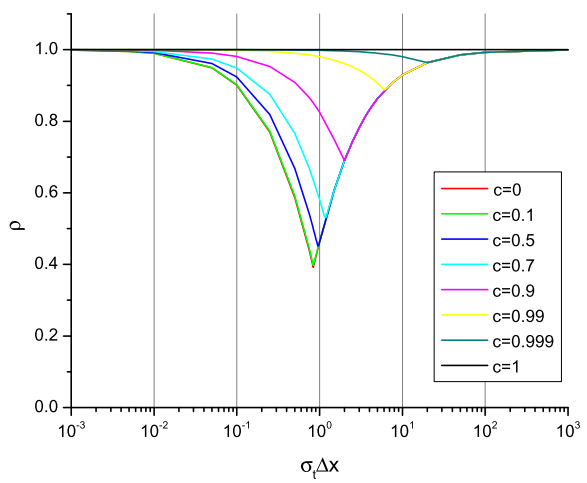

(b) $S_{8}$

Fig. 1. Theoretically estimated spectral radii for problems in 1D slab geometry.

for any scattering ratio $c$. The values of spectral radius for $S_{2}$ problems versus $\sigma_{t} \Delta x$ for some set of $c$ are plotted in Fig. 1(a). We see that the spectral radius $\rho \leq 1$. The minimum value is $\rho=0$. It occurs in problems without scattering $(c=0)$ for $\sigma_{t} \Delta x=\frac{2}{\sqrt{3}}$. For this value of optical thickness of mesh intervals the Pade approximation of the exponential attenuation factor in the DD scheme equals zero. The DD solution becomes independent of the angular flux incoming from neighboring spatial intervals. As a result ITMM generates the numerical solution without iterations.

We now demonstrate results of Fourier analysis for a more general case, namely, $S_{8}$ problems. Fig. 1(b) shows theoretically estimated spectral radii for $S_{8}$ Gauss-Legendre quadrature set. The value of spectral radius depends on $c$ and $\sigma_{t} \Delta x$ in a way similar to the $S_{2}$ case. The convergence of ITMM slows down with increase in optical thickness of mesh intervals as well as with its decrease. The minimum value of the spectral radius $\rho \approx 0.392$ is reached for $c=0$ and $\sigma_{t} \Delta x \approx 0.839$. Thus the Fourier analysis of infinite-medium problems revealed that ITMM can converge in 1D transport problems. The analysis also showed that it loses its effectiveness $(\rho \rightarrow 1)$ for any $c \geq 0$ only as $\sigma_{t} \Delta x \rightarrow \infty$ or $\sigma_{t} \Delta x \rightarrow 0$.

\section{Analysis of problems in 2D Cartesian geometry}

\subsection{Formulation of the computational method}

The steady-state one-group transport problem in case of 2D Cartesian geometry has the following form:

$$
\begin{aligned}
& \Omega_{x} \frac{\partial}{\partial x} \psi(\mathbf{r}, \boldsymbol{\Omega})+\Omega_{y} \frac{\partial}{\partial y} \psi(\mathbf{r}, \boldsymbol{\Omega})+\sigma_{t}(\mathbf{r}) \psi(\mathbf{r}, \boldsymbol{\Omega})=\frac{1}{4 \pi}\left(\sigma_{s}(\mathbf{r}) \phi(\mathbf{r})+q(\mathbf{r})\right) \quad \mathbf{r} \in \mathcal{D}, \\
& \mathcal{D}=\{0<x<X, 0<y<Y\} \\
& \left.\psi\right|_{\mathbf{r} \in \partial \mathcal{D}}=\psi_{\text {in }} \text { for } \boldsymbol{\Omega} \cdot \mathbf{e}_{n}<0, \\
& \phi(\mathbf{r})=\int_{4 \pi} \psi(\mathbf{r}, \boldsymbol{\Omega}) d \Omega .
\end{aligned}
$$

Here $\mathbf{r}$ is the spatial position, $\boldsymbol{\Omega}=\left(\Omega_{x}, \Omega_{y}\right)$ is the unit vector in the direction of particle motion, $\mathbf{e}_{n}$ is the outward normal at the boundary surface, $\psi_{\text {in }}$ is the angular flux of incoming particles. The DD scheme for approximation of the transport equation (37) is defined as follows [15]:

$$
\begin{aligned}
& \frac{\left|\Omega_{x, m}\right|}{\Delta x_{k}}\left(\psi_{m, k, j}^{x+}-\psi_{m, k, j}^{x-}\right)+\frac{\left|\Omega_{y, m}\right|}{\Delta y_{j}}\left(\psi_{m, k, j}^{y+}-\psi_{m, k, j}^{y-}\right)+\sigma_{t, k, j} \psi_{m, k, j}=\frac{1}{4 \pi}\left(\sigma_{s, k, j} \phi_{k, j}+q_{k, j}\right), \\
& \psi_{m, k, j}=\frac{1}{2}\left(\psi_{m, k, j}^{x+}+\psi_{m, k, j}^{x-}\right), \\
& \psi_{m, k, j}=\frac{1}{2}\left(\psi_{m, k, j}^{y+}+\psi_{m, k, j}^{y-}\right), \\
& \phi_{k, j}=\sum_{m=1}^{M} \psi_{m, k, j} w_{m}, \\
& m=1, \ldots, M, \quad k=1, \ldots, N_{x}, \quad j=1, \ldots, N_{y}, \\
& \psi_{m, k, j}^{x \pm}=\psi_{m, k \pm 1 / 2, j}, \text { for } \Omega_{x, m}>0, \quad \psi_{m, k, j}^{x \pm}=\psi_{m, k \mp 1 / 2, j}, \text { for } \Omega_{x, m}<0, \\
& \psi_{m, k, j}^{y \pm}=\psi_{m, k, j \pm 1 / 2}, \text { for } \Omega_{y, m}>0, \quad \psi_{m, k, j}^{y \pm}=\psi_{m, k, j \mp 1 / 2}, \text { for } \Omega_{y, m}<0 .
\end{aligned}
$$


Here $k$ and $j$ are indices of spatial intervals in $x$ and $y$, respectively, $\psi_{m, k, j}$ is the $m$-th angular flux averaged over the $(k, j)$-cell, $\psi_{m, k \pm 1 / 2, j}$ and $\psi_{m, k, j \pm 1 / 2}$ are face-average $m$-th angular fluxes, $\sigma_{t, k, j}, \sigma_{s, k, j}, q_{k, j}$ are cell-average values of the total and scattering cross sections and the source, respectively, $\Delta x_{k}$ and $\Delta y_{j}$ are width and height of the $(k, j)$-cell. The ITMM applied to Eqs. (40) leads to the block Jacobi iteration scheme defined as follows:

$$
\begin{aligned}
& \frac{\left|\Omega_{x, m}\right|}{\Delta x_{k}}\left(\psi_{m, k, j}^{x+(s)}-\psi_{m, k, j}^{x-(s-1)}\right)+\frac{\left|\Omega_{y, m}\right|}{\Delta y_{j}}\left(\psi_{m, k, j}^{y+(s)}-\psi_{m, k, j}^{y-(s-1)}\right)+\sigma_{t, k, j} \psi_{m, k, j}^{(s)}=\frac{1}{4 \pi}\left(\sigma_{s, k, j} \phi_{k, j}^{(s)}+q_{k, j}\right), \\
& \psi_{m, k, j}^{(s)}=\frac{1}{2}\left(\psi_{m, k, j}^{x+(s)}+\psi_{m, k, j}^{x-(s-1)}\right), \\
& \psi_{m, k, j}^{(s)}=\frac{1}{2}\left(\psi_{m, k, j}^{y+(s)}+\psi_{m, k, j}^{y-(s-1)}\right), \\
& \phi_{k, j}^{(s)}=\sum_{m=1}^{M} \psi_{m, k, j}^{(s)} w_{m} .
\end{aligned}
$$

We subtract Eqs. (42) from the corresponding Eqs. (40) to obtain the following equations for the iterative errors:

$$
\begin{aligned}
& v_{x, m, k, j}\left(\delta \psi_{m, k, j}^{x+(s)}-\delta \psi_{m, k, j}^{x-(s-1)}\right)+v_{y, m, k, j}\left(\delta \psi_{m, k, j}^{y+(s)}-\delta \psi_{m, k, j}^{y-(s-1)}\right)+\delta \psi_{m, k, j}^{(s)}=\frac{c_{k, j}}{4 \pi} \delta \phi_{k, j}^{(s)}, \\
& \delta \psi_{m, k, j}^{(s)}=\frac{1}{2}\left(\delta \psi_{m, k, j}^{x+(s)}+\delta \psi_{m, k, j}^{x-(s-1)}\right), \\
& \delta \psi_{m, k, j}^{(s)}=\frac{1}{2}\left(\delta \psi_{m, k, j}^{y+(s)}+\delta \psi_{m, k, j}^{y-(s-1)}\right), \\
& \delta \phi_{k, j}^{(s)}=\sum_{m=1}^{M} \delta \psi_{m, k, j}^{(s)} w_{m}, \\
& \delta \psi_{m, k, j}^{x+(s)}=\delta \psi_{m, k \pm 1 / 2, j}^{(s)}, \text { for } \Omega_{x, m} \gtrless 0, \quad \delta \psi_{m, k, j}^{x-(s-1)}=\delta \psi_{m, k \mp 1 / 2, j}^{(s-1)}, \text { for } \Omega_{x, m} \gtrless 0, \\
& \delta \psi_{m, k, j}^{y+(s)}=\delta \psi_{m, k, j \pm 1 / 2}^{(s)}, \text { for } \Omega_{y, m} \gtrless 0, \quad \delta \psi_{m, k, j}^{y-(s-1)}=\delta \psi_{m, k, j \mp 1 / 2}^{(s-1)}, \text { for } \Omega_{y, m} \gtrless 0,
\end{aligned}
$$

where we also defined

$$
v_{x, m, k, j}=\frac{\left|\Omega_{x, m}\right|}{\sigma_{t, k, j} \Delta x_{k}}, \quad v_{y, m, k, j}=\frac{\left|\Omega_{y, m}\right|}{\sigma_{t, k, j} \Delta y_{j}}, \quad c_{k, j}=\frac{\sigma_{s, k, j}}{\sigma_{t, k, j}} .
$$

Using equations (43) to eliminate the cell-averaged quantities leads to the following equations for the iterative errors in the face-averaged angular fluxes at two consecutive iteration levels:

$$
\begin{aligned}
& p_{x, m, k, j}^{+} \delta \psi_{m, k, j}^{x+(s)}+p_{x, m, k, j}^{-} \delta \psi_{m, k, j}^{x-(s-1)}+p_{y, m, k, j}^{+} \delta \psi_{m, k, j}^{y+(s)}+p_{y, m, k, j}^{-} \delta \psi_{m, k, j}^{y-(s-1)} \\
& =\frac{c_{k, j}}{4 \pi} \sum_{m^{\prime} \neq m}\left(\delta \psi_{m^{\prime}, k, j}^{x+(s)}+\delta \psi_{m^{\prime}, k, j}^{x-(s-1)}+\delta \psi_{m^{\prime}, k, j}^{y+(s)}+\delta \psi_{m^{\prime}, k, j}^{y-(s-1)}\right) w_{m^{\prime}}, \\
& \delta \psi_{m, k, j}^{x+(s)}+\delta \psi_{m, k, j}^{x-(s-1)}=\delta \psi_{m, k, j}^{y+(s)}+\delta \psi_{m, k, j}^{y-(s-1)},
\end{aligned}
$$

where

$$
p_{\alpha, m, k, j}^{ \pm}=1 \pm 4 v_{\alpha, m, k, j}-\frac{c_{k, j} w_{m}}{4 \pi}, \quad \alpha=x, y .
$$

Another form of ITMM equations can be derived if one uses the auxiliary conditions (40b) and (40c) to eliminate the outgoing angular fluxes, $\psi_{m, k, j}^{\alpha+}(\alpha=x, y)$, in favor of the cell-averaged angular flux in Eq. (40a) and integrate the resulting form of the detailed balance equation over discrete angular directions to get the cell-average scalar flux in terms of face-average angular fluxes on incoming faces:

$$
\phi_{k, j}=\sum_{m=1}^{M}\left(\gamma_{m, k, j}^{x} \psi_{m, k, j}^{x-}+\gamma_{m, k, j}^{y} \psi_{m, k, j}^{y-}\right)+\frac{q_{k, j} \eta_{k, j}}{4 \pi \sigma_{t, k, j}} \sum_{m=1}^{M} \beta_{m, k, j},
$$

where

$$
\begin{aligned}
& \gamma_{m, k, j}^{x}=2 \eta_{k, j} v_{x, m, k, j} \beta_{m, k, j}, \quad \gamma_{m, k, j}^{y}=2 \eta_{k, j} v_{y, m, k, j} \beta_{m, k, j}, \\
& \beta_{m, k, j}=\frac{w_{m}}{1+2\left(v_{x, m, k, j}+v_{y, m, k, j}\right)}, \\
& \eta_{k, j}=\left[1-\frac{c_{k, j}}{4 \pi} \sum_{m=1}^{M} \beta_{m, k, j}\right]^{-1} .
\end{aligned}
$$


This form of the cell-average scalar flux is applied to formulate equations of the ITMM in the response-matrix form (7). It is used below in our analysis.

\subsection{Fourier analysis of infinite-medium transport problems in $2 D$}

To perform Fourier analysis of the ITMM with the PBJ algorithm we consider problems with infinite homogeneous medium and uniform spatial grid ( $\Delta x_{k}=\Delta x$ and $\Delta y_{j}=\Delta y$ ). The equations for the iterative errors (46) become

$$
\begin{aligned}
& p_{m, x}^{+} \delta \psi_{m, k, j}^{x+(s)}+p_{m, x}^{-} \delta \psi_{m, k, j}^{x-(s-1)}+p_{m, y}^{+} \delta \psi_{m, k, j}^{y+(s)}+p_{m, y}^{-} \delta \psi_{m, k, j}^{y-(s-1)} \\
& =\sum_{m^{\prime} \neq m} \tilde{c}_{m^{\prime}}\left(\delta \psi_{m^{\prime}, k, j}^{x+(s)}+\delta \psi_{m^{\prime}, k, j}^{x-(s-1)}+\delta \psi_{m^{\prime}, k, j}^{y+(s)}+\delta \psi_{m^{\prime}, k, j}^{y-(s-1)}\right) \\
& \delta \psi_{m, k, j}^{x+(s)}+\delta \psi_{m, k, j}^{x-(s-1)}=\delta \psi_{m, k, j}^{y+(s)}+\delta \psi_{m, k, j}^{y-(s-1)},
\end{aligned}
$$

where

$$
\tilde{c}_{m}=\frac{c w_{m}}{4 \pi}, \quad p_{m, \alpha}^{ \pm}=1 \pm 4 v_{m}^{\alpha}-\tilde{c}_{m}, \quad v_{m}^{\alpha}=\frac{\left|\Omega_{\alpha, m}\right|}{\sigma_{t} \Delta \alpha}, \quad \alpha=x, y .
$$

We introduce a Fourier ansatz:

$$
\begin{aligned}
& \delta \psi_{m, k+1 / 2, j}^{(s)}=\omega^{s}(\lambda) a_{x, m}(\lambda) e^{i \sigma_{t}\left(\lambda_{x} x_{k+1 / 2}+\lambda_{y} y_{j}\right)}, \\
& \delta \psi_{m, k, j+1 / 2}^{(s)}=\omega^{s}(\lambda) a_{y, m}(\lambda) e^{i \sigma_{t}\left(\lambda_{x} x_{k}+\lambda_{y} y_{j+1 / 2}\right)}
\end{aligned}
$$

for a Fourier error mode with an arbitrary $\lambda=\left(\lambda_{x}, \lambda_{y}\right)$. Substituting (54) in Eqs. (52) and taking into account Eqs. (44) we obtain the equations

$$
\left(\mathbf{T}^{+}\right)^{-1} \mathbf{T}^{-} \boldsymbol{a}=\omega \mathbf{I a}
$$

for $\omega$ and the vector of coefficients of the Fourier expansion (54)

$$
\boldsymbol{a}=\left(a_{x, 1}, \ldots, a_{x, M}, a_{y, 1}, \ldots, a_{y, M}\right)^{T},
$$

where the matrices are defined as follows:

$$
\begin{aligned}
& \mathbf{T}^{+}=\left(\begin{array}{cc}
\mathbf{P}_{x}^{+} \mathbf{E}_{x}^{+} & \mathbf{P}_{y}^{+} \mathbf{E}_{y}^{+} \\
\mathbf{E}_{x}^{+} & -\mathbf{E}_{y}^{+}
\end{array}\right), \\
& \mathbf{T}^{-}=(-1)\left(\begin{array}{cc}
\mathbf{P}_{x}^{-} \mathbf{E}_{x}^{-} & \mathbf{P}_{y}^{-} \mathbf{E}_{y}^{-} \\
\mathbf{E}_{x}^{-} & -\mathbf{E}_{y}^{-}
\end{array}\right), \\
& \mathbf{P}_{\alpha}^{ \pm}=\left(\begin{array}{ccccccc}
p_{\alpha, 1}^{ \pm} & -\tilde{c}_{2} & \ldots & & & & -\tilde{c}_{M} \\
-\tilde{c}_{1} & p_{\alpha, 2}^{ \pm} & -\tilde{c}_{3} & \ldots & & & -\tilde{c}_{M} \\
\vdots & & \ddots & & & & \vdots \\
-\tilde{c}_{1} & \ldots & -\tilde{c}_{m-1} & p_{\alpha, m}^{ \pm} & -\tilde{c}_{m+1} & \ldots & -\tilde{c}_{M} \\
\vdots & & & & \ddots & & \vdots \\
-\tilde{c}_{1} & \ldots & & & -\tilde{c}_{M-2} & p_{\alpha, M-1}^{ \pm} & -\tilde{c}_{M} \\
-\tilde{c}_{1} & \ldots & & & & -\tilde{c}_{M-1} & p_{\alpha, M}^{ \pm}
\end{array}\right), \\
& \mathbf{E}_{\alpha}^{ \pm}=\operatorname{diag}\left(e^{i \tau_{\alpha, 1}^{ \pm}}, \ldots, e^{i \tau_{\alpha, M}^{ \pm}}\right), \\
& \tau_{\alpha, m}^{ \pm}=\frac{1}{2} \varkappa_{\alpha, m}^{ \pm} \tilde{\lambda}_{\alpha} \\
& \tilde{\lambda}_{\alpha}=\lambda_{\alpha} \sigma_{t} \Delta \alpha \text {, } \\
& \varkappa_{\alpha, m}^{ \pm}= \begin{cases} \pm 1 & \text { for } \Omega_{\alpha, m}>0, \\
\mp 1 & \text { for } \Omega_{\alpha, m}<0 .\end{cases}
\end{aligned}
$$

We note that $\omega$ is an eigenvalue of the matrix $\left(\mathbf{T}^{+}\right)^{-1} \mathbf{T}^{-}$with dimensionality $2 M \times 2 M$. The spectral radius is defined by

$$
\rho=\sup _{\lambda_{x}, \lambda_{y}}\left|\omega\left(\lambda_{x}, \lambda_{y}\right)\right|
$$




\section{Fourier analysis results in $2 \mathrm{D}$}

\subsection{Analytical results of Fourier analysis of $S_{2}$ case in $2 D$} by

In this section we consider $S_{2}$ problems. There are four discrete directions $(M=4)$ and the $S_{2}$ quadrature set is given

$$
w_{m}=\pi, \quad\left|\Omega_{m, x}\right|=\left|\Omega_{m, y}\right|=\frac{1}{\sqrt{3}}, \quad m=1, \ldots, 4 .
$$

The matrices $\mathbf{T}^{ \pm}$are $8 \times 8$ and defined by $\mathbf{P}_{\alpha}^{ \pm}$and $\mathbf{E}_{\alpha}^{ \pm}$of the following form:

$$
\begin{aligned}
& \mathbf{P}_{\alpha}^{ \pm}=\left(\begin{array}{cccc}
\bar{p}_{\alpha}^{ \pm} & -\bar{c} & -\bar{c} & -\bar{c} \\
-\bar{c} & \bar{p}_{\alpha}^{ \pm} & -\bar{c} & -\bar{c} \\
-\bar{c} & -\bar{c} & \bar{p}_{\alpha}^{ \pm} & -\bar{c} \\
-\bar{c} & -\bar{c} & -\bar{c} & \bar{p}_{\alpha}^{ \pm}
\end{array}\right), \\
& \mathbf{E}_{x}^{ \pm}=\operatorname{diag}\left(e^{ \pm i \tau_{x}}, e^{\mp i \tau_{x}}, e^{\mp i \tau_{x}}, e^{ \pm i \tau_{x}}\right), \\
& \mathbf{E}_{y}^{ \pm}=\operatorname{diag}\left(e^{ \pm i \tau_{y}}, e^{ \pm i \tau_{y}}, e^{\mp i \tau_{y}}, e^{\mp i \tau_{y}}\right),
\end{aligned}
$$

where

$$
\bar{c}=\frac{1}{4} c, \quad \bar{p}_{\alpha}^{ \pm}=1 \pm 4 \bar{v}_{\alpha}-\bar{c}, \quad \bar{v}_{\alpha}=\frac{1}{\sqrt{3} \sigma_{t} \Delta \alpha}, \quad \alpha=x, y .
$$

First we analyze eigenvalues and eigenvectors. The analysis of the equation (55) for $S_{2}$ case shows that if $\tilde{\lambda}_{x}=\tilde{\lambda}_{y}=\tilde{\lambda}_{\text {, }}$ then a subset of eigenvalues is given by

$$
\omega=-e^{ \pm i \tilde{\lambda}}
$$

and hence $|\omega|=1$. This means that these error modes are not reduced during iterations. For a particular case of a spatially constant Fourier mode that corresponds to $\tilde{\lambda}_{x}=\tilde{\lambda}_{y}=0$, the eigenvalue $\omega=-1$ has algebraic multiplicity 4 . If $\tilde{\lambda}_{x}=\tilde{\lambda}_{y}=\pi$, then $\omega=1$ is the eigenvalue with algebraic multiplicity 4 as well. These results do not depend on values of the scattering ratio $c$, total cross section $\sigma_{t}$ and cell sizes $\Delta x$ and $\Delta y$. Further analysis of $\omega$ as a function of $\lambda$ for various values of $c$ and optical thickness of cells showed that it doesn't exceed 1 and hence the spectral radius $\rho=1$ for the $S_{2}$ case.

The transport solution $\psi$ in an infinite homogeneous medium is spatially constant and does not depend on angular variable $\boldsymbol{\Omega}_{m}$, i.e. it is an isotropic function. Hence this is the form of the solution the iterative method converges to. Thus it is interesting to study spatially constant and isotropic error modes. The analysis shows that the eigenvector corresponding to spatially constant mode $\left(\tilde{\lambda}_{x}=\tilde{\lambda}_{y}=0\right)$ meets the following condition

$$
\bar{v}_{x} a_{x, l}+\bar{v}_{y} a_{y, l}=0, l=1, \ldots, 4
$$

and its components depend on the discrete ordinate directions, i.e. this mode is not isotropic. The isotropic eigenvector $\left(a_{x, l}=a_{y, l}=\right.$ const, $\left.l=1, \ldots, 4\right)$ is associated with the following eigenvalues:

$$
\omega=-\omega_{\text {iso }} \text { for } \tilde{\lambda}_{x}=\tilde{\lambda}_{y}=0, \quad \omega=\omega_{\text {iso }} \text { for } \tilde{\lambda}_{x}=\tilde{\lambda}_{y}=\pi,
$$

where

$$
\omega_{i s o}=\frac{1-c-2\left(\bar{v}_{x}+\bar{v}_{y}\right)}{1-c+2\left(\bar{v}_{x}+\bar{v}_{y}\right)} .
$$

Thus, these eigenmodes are associated with $\left|\omega_{\text {iso }}\right|<1$ for $c \neq 1$ and arbitrary spatial cells. $\omega_{\text {iso }}$ has the following limit for any $c$ :

$$
\begin{aligned}
& \omega_{\text {iso }} \rightarrow 1 \text { as } \sigma_{t} \Delta x \rightarrow \infty \text { and } \sigma_{t} \Delta y \rightarrow \infty, \\
& \omega_{\text {iso }} \rightarrow-1 \text { as } \sigma_{t} \Delta x \rightarrow 0 \text { and } \sigma_{t} \Delta y \rightarrow 0 .
\end{aligned}
$$

To demonstrate more details of theoretical results we present eigenvalues $\omega$ computed for selected wave numbers in case of low and high scattering ratios. Table 1 shows eigenvalues $\omega$ computed for problems with $c=0.1, \sigma_{t}=1$, and $\Delta x=\Delta y=1$. The eigenvalues for high scattering ratio $c=0.999$ are listed in Table 2 . We note that the isotropic eigenvector is associated with $\omega_{5}$ for $\tilde{\lambda}_{x}=\tilde{\lambda}_{y}=0$ and $\tilde{\lambda}_{x}=\tilde{\lambda}_{y}=\pi$.

We performed calculations of numerical test problems that simulated infinite homogeneous medium. Those are problems in a finite domain $\mathcal{D}$ with reflective boundary conditions defined by:

$$
\left.\psi(\mathbf{r}, \boldsymbol{\Omega})\right|_{\mathbf{r} \in \partial \mathcal{D}}=\left.\psi\left(\mathbf{r}, \boldsymbol{\Omega}^{*}\right)\right|_{\mathbf{r} \in \partial \mathcal{D}} \quad \text { for } \quad \boldsymbol{\Omega} \cdot \mathbf{e}_{n}<0, \quad \mathbf{e}_{n} \cdot \boldsymbol{\Omega}=-\mathbf{e}_{n} \cdot \boldsymbol{\Omega}^{*}, \quad \mathbf{e}_{n} \cdot \boldsymbol{\Omega} \times \boldsymbol{\Omega}^{*}=0 .
$$


Table 1

Eigenvalues $\omega_{k}$ for $S_{2}, c=0.1, \sigma_{t} \Delta x=\sigma_{t} \Delta y=1\left(\tilde{\lambda}_{\alpha}=\lambda_{\alpha} \sigma_{t} \Delta \alpha\right)$.

\begin{tabular}{|c|c|c|c|c|c|}
\hline$\left[\tilde{\lambda}_{x}, \tilde{\lambda}_{y}\right]$ & {$[0,0]$} & {$\left[0, \frac{\pi}{2}\right]$} & {$\left[\frac{\pi}{5}, \frac{\pi}{5}\right]$} & {$\left[\frac{\pi}{2}, \frac{\pi}{2}\right]$} & {$[\pi, \pi]$} \\
\hline$\omega_{1}$ & -1 & $-0.6250-i 0.6252$ & $-0.8090-i 0.5878$ & $i$ & 1 \\
\hline$\omega_{2}$ & -1 & $-0.6250+i 0.6252$ & $-0.8090+i 0.5878$ & $-i$ & 1 \\
\hline$\omega_{3}$ & -1 & $-0.6208-i 0.6208$ & -0.9233 & -0.6458 & 1 \\
\hline$\omega_{4}$ & -1 & $-0.6208+i 0.6208$ & -0.9193 & 0.6458 & 1 \\
\hline$\omega_{5}$ & 0.4391 & $0.3337-i 0.3331$ & 0.4518 & 0.6290 & -0.4391 \\
\hline$\omega_{6}$ & 0.3957 & $0.3337+i 0.3331$ & 0.4304 & -0.6290 & -0.3957 \\
\hline$\omega_{7}$ & 0.3957 & $0.3187+i 0.3187$ & $0.3290+i 0.2378$ & $-i 0.4060$ & -0.3957 \\
\hline$\omega_{8}$ & 0.3957 & $0.3187-i 0.3187$ & $0.3290-i 0.2378$ & $i 0.4060$ & -0.3957 \\
\hline
\end{tabular}

Table 2

Eigenvalues $\omega_{k}$ for $S_{2}, c=0.999, \sigma_{t} \Delta x=\sigma_{t} \Delta y=1\left(\tilde{\lambda}_{\alpha}=\lambda_{\alpha} \sigma_{t} \Delta \alpha\right)$.

\begin{tabular}{|c|c|c|c|c|c|}
\hline$\left[\tilde{\lambda}_{x}, \tilde{\lambda}_{y}\right]$ & {$[0,0]$} & {$\left[0, \frac{\pi}{2}\right]$} & {$\left[\frac{\pi}{5}, \frac{\pi}{5}\right]$} & {$\left[\frac{\pi}{2}, \frac{\pi}{2}\right]$} & {$[\pi, \pi]$} \\
\hline$\omega_{1}$ & -1 & $-0.6571-i 0.6704$ & $-0.8090-i 0.5878$ & $i$ & 1 \\
\hline$\omega_{2}$ & -1 & $-0.6571+i 0.6704$ & $-0.8090+i 0.5878$ & $-i$ & 1 \\
\hline$\omega_{3}$ & -1 & $-0.6208-i 0.6208$ & -0.9595 & -0.8117 & 1 \\
\hline$\omega_{4}$ & -1 & $-0.6208+i 0.6208$ & -0.9193 & 0.8117 & 1 \\
\hline$\omega_{5}$ & 0.9991 & $0.5058-i 0.4390$ & 0.8232 & 0.6290 & -0.9991 \\
\hline$\omega_{6}$ & 0.3957 & $0.5058+i 0.4390$ & 0.4304 & -0.6290 & -0.3957 \\
\hline$\omega_{7}$ & 0.3957 & $0.3187+i 0.3187$ & $0.3879+i 0.2181$ & $-i 0.4872$ & -0.3957 \\
\hline$\omega_{8}$ & 0.3957 & $0.3187-i 0.3187$ & $0.3879-i 0.2181$ & $i 0.4872$ & -0.3957 \\
\hline
\end{tabular}

In some of this kind of test problems we observed that the cell-average scalar flux corresponding to error modes associated with $|\omega|=1$ converges. This initiated analysis of convergence behavior of scalar fluxes presented below.

We now analyze iterative error modes of $\phi_{k, j}$. A single Fourier error mode of the cell-average angular flux can be written as

$$
\delta \psi_{m, k, j}^{(s)}=\omega^{s}(\lambda) b_{m}(\lambda) e^{i \sigma_{t}\left(\lambda_{x} x_{k}+\lambda_{y} y_{j}\right)} .
$$

Then, the corresponding error mode of the cell-average scalar flux is given by

$$
\delta \phi_{k, j}^{(s)}=\omega^{s}(\lambda) b(\lambda) e^{i \sigma_{t}\left(\lambda_{x} x_{k}+\lambda_{y} y_{j}\right)},
$$

where

$$
b(\lambda)=\sum_{m} b_{m}(\lambda) w_{m} .
$$

On the other hand, using Eq. (48) the error in $s$-th iterate of the cell-average scalar flux for the ITMM can be written in terms of errors in incoming face-average angular fluxes as follows:

$$
\delta \phi_{k, j}^{(s)}=\sum_{m}\left(\gamma_{x} \delta \psi_{m, k, j}^{x-(s-1)}+\gamma_{y} \delta \psi_{m, k, j}^{y-(s-1)}\right),
$$

where

$$
\gamma_{\alpha}=\frac{2 \pi \eta \bar{v}_{\alpha}}{1+2\left(\bar{v}_{x}+\bar{v}_{y}\right)}, \quad \eta=\left[1-\frac{c}{1+2\left(\bar{v}_{x}+\bar{v}_{y}\right)}\right]^{-1} .
$$

This leads to the following formula

$$
\begin{aligned}
\delta \phi_{k, j}^{(s)}= & e^{i \sigma_{t}\left(\lambda_{x} x_{k}+\lambda_{y} y_{j}\right)} \omega^{s-1}\left[\gamma_{x}\left(\left(a_{x, 1}+a_{x, 4}\right) e^{-i \tau_{x}}+\left(a_{x, 2}+a_{x, 3}\right) e^{i \tau_{x}}\right)\right. \\
& \left.+\gamma_{y}\left(\left(a_{y, 1}+a_{y, 2}\right) e^{-i \tau_{y}}+\left(a_{y, 3}+a_{y, 4}\right) e^{i \tau_{y}}\right)\right]
\end{aligned}
$$

in terms of coefficients of Fourier expansion (54) for errors in the face-average angular fluxes.

First we consider the case of spatially constant error modes $\left(\tilde{\lambda}_{x}=\tilde{\lambda}_{y}=0\right)$ which corresponds to $\omega=-1$. The equation (82) gives rise to

$$
\delta \phi_{k, j}^{(s)}=e^{i \sigma_{t}\left(\lambda_{x} x_{k}+\lambda_{y} y_{j}\right)}(-1)^{s-1} \gamma_{x}\left[\sum_{m=1}^{4} a_{x, m}+\frac{\bar{v}_{y}}{\bar{v}_{x}} \sum_{m=1}^{4} a_{y, m}\right] .
$$




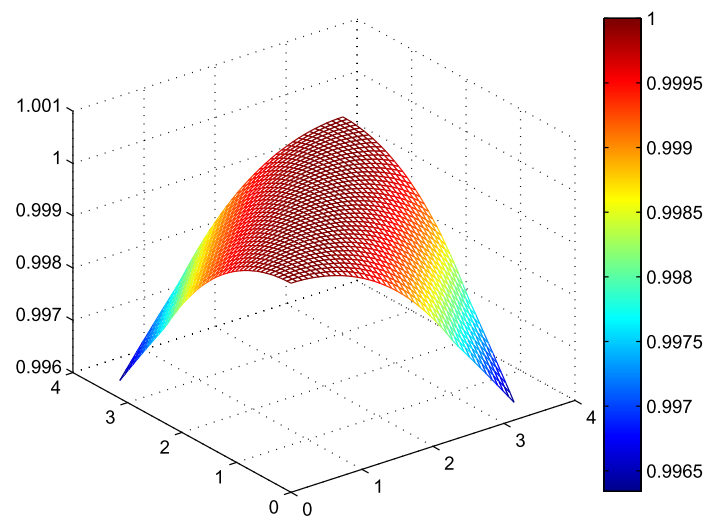

(a) $\sigma_{t} \Delta x=\sigma_{t} \Delta y=0.01$

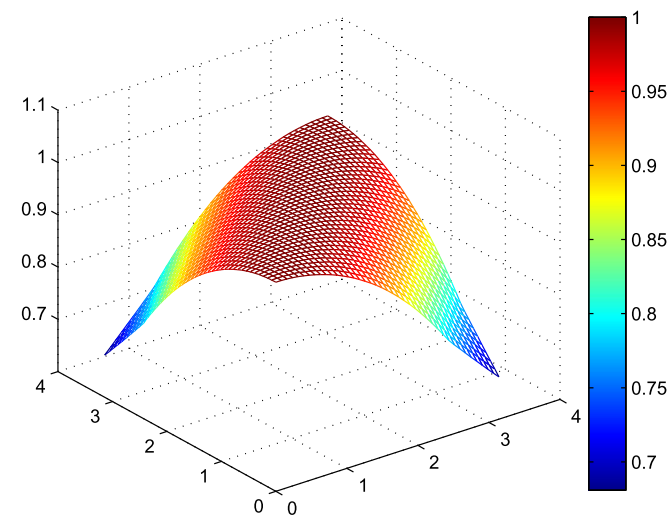

(b) $\sigma_{t} \Delta x=\sigma_{t} \Delta y=1$

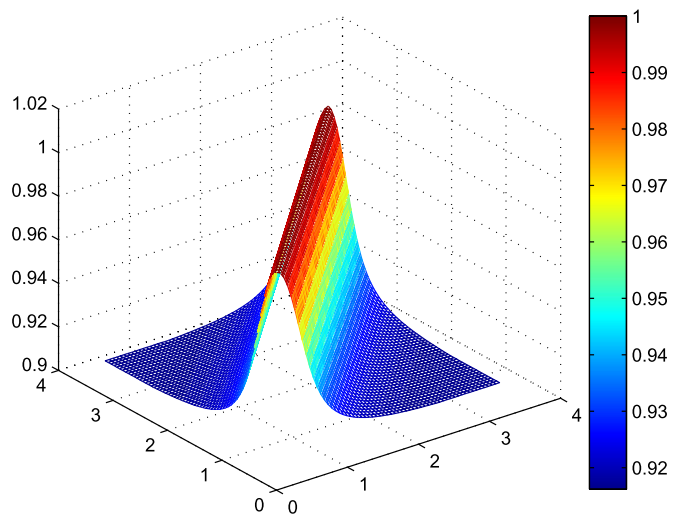

(c) $\sigma_{t} \Delta x=\sigma_{t} \Delta y=10$

Fig. 2. $\max _{k}\left|\omega_{k}\left(\tilde{\lambda}_{x}, \tilde{\lambda}_{y}\right)\right|$ for $S_{8}$ problems in $2 \mathrm{D}$ with $c=0$.

As it shown above components of the associated eigenvector satisfy Eq. (71). Taking into account this relationship in Eq. (83) we get

$$
\delta \phi_{k, j}^{(s)}=0
$$

and hence $\left.b\right|_{\lambda=(0,0)}=0$ for an arbitrary rectangular grid. This means the spatially flat errors modes in the cell-average scalar fluxes are eliminated in one iteration in spite of the fact that $|\omega|=1$. Note that the amplitude of the corresponding error mode in the face-average angular fluxes does not change during iterations.

The case of the mode with $\tilde{\lambda}_{x}=\tilde{\lambda}_{y}=\pi$ is similar. The corresponding eigenvalue $\omega$ is equal to 1 and Eq. (82) results in (83). The associated eigenvectors also meet the relation (71) and hence we get that the errors for the cell-average scalar leads to (84). We now analyze the error mode with $\tilde{\lambda}_{x}=\tilde{\lambda}_{y}=\frac{\pi}{2}$ for which $\omega= \pm i$. In this case we get

$$
\begin{aligned}
\delta \phi_{k, j}^{(s)}= & e^{i \sigma_{t}\left(\lambda_{x} x_{k}+\lambda_{y} y_{j}\right)}( \pm i)^{s-1} \frac{\gamma_{x}}{\sqrt{2}}\left[\left(\left(a_{x, 1}+a_{x, 4}\right)(1-i)+\left(a_{x, 2}+a_{x, 3}\right)(1+i)\right)\right. \\
& \left.+\frac{\bar{v}_{y}}{\bar{v}_{x}}\left(\left(a_{y, 1}+a_{y, 2}\right)(1-i)+\left(a_{y, 3}+a_{y, 4}\right)(1+i)\right)\right]
\end{aligned}
$$

The components of associated eigenvectors meet the following conditions:

$$
\begin{array}{llll}
\bar{v}_{x} a_{x, 1}+\bar{v}_{y} a_{y, 1}=0, & a_{x, l}=a_{y, l}=0, & l=2,3,4, & \text { for } \quad \omega=i, \\
\bar{v}_{x} a_{x, 3}+\bar{v}_{y} a_{y, 3}=0, & a_{x, l}=a_{y, l}=0, & l=1,2,4, & \text { for } \quad \omega=-i .
\end{array}
$$

Using Eq. (86) for $\omega=i$ and Eq. (87) for $\omega=-i$ in Eq. (85), we conclude that in each of these cases $\delta \phi_{k, j}^{(s)}=0$ and $\left.b\right|_{\lambda=\left(\frac{\pi}{2}, \frac{\pi}{2}\right)}=0$ for arbitrary rectangular cells.

The presented analysis shows that convergence behavior of the scalar flux in considered cases is different from that of the angular flux. The amplitude of error modes of the face-average angular fluxes will not change because modulus of the 


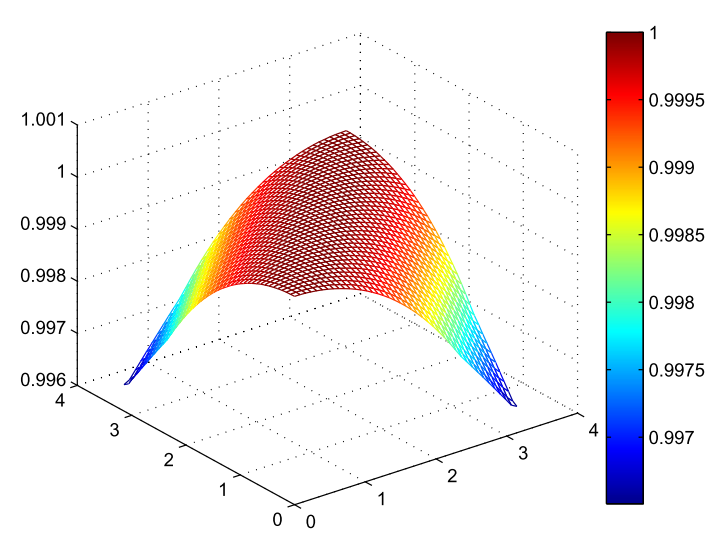

(a) $\sigma_{t} \Delta x=\sigma_{t} \Delta y=0.01$

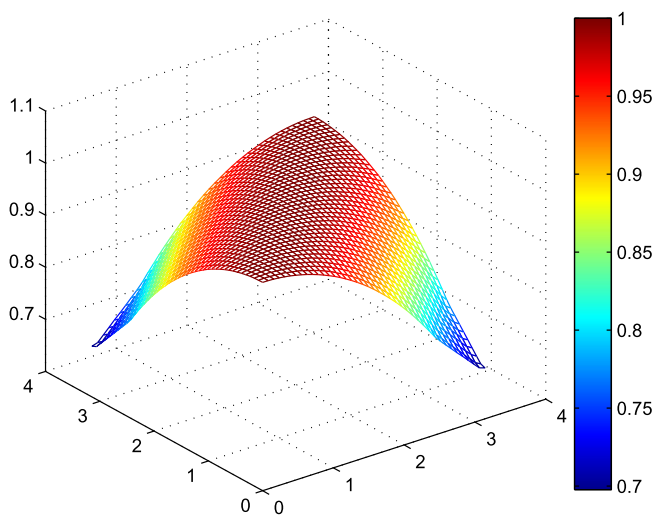

(b) $\sigma_{t} \Delta x=\sigma_{t} \Delta y=1$

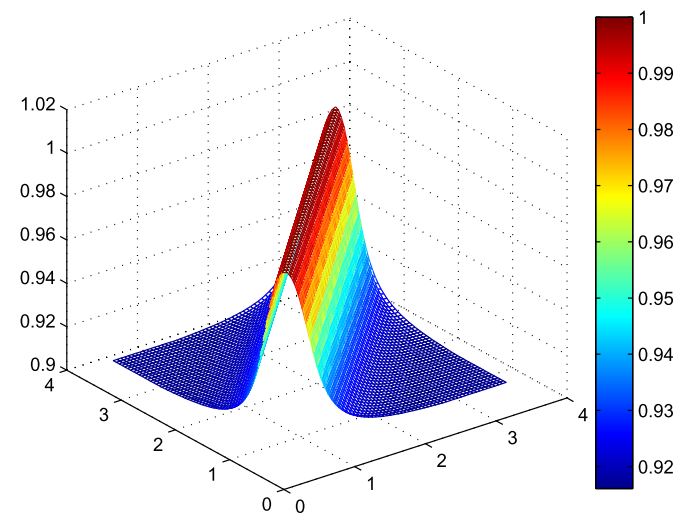

(c) $\sigma_{t} \Delta x=\sigma_{t} \Delta y=10$

Fig. 3. $\max _{k}\left|\omega_{k}\left(\tilde{\lambda}_{x}, \tilde{\lambda}_{y}\right)\right|$ for $S_{8}$ problems in $2 \mathrm{D}$ with $c=0.5$.

eigenvalue equals one. However, the corresponding error modes of the cell-average scalar flux will turn to zero because the modes of the angular flux contributing to the iterative errors of the scalar flux cancel out due to superposition. This effect was observed in numerical tests with reflective boundary conditions described above.

\subsection{Fourier analysis results for $S_{N}$ case in $2 D$}

We now consider $S_{8}$ discrete ordinate problems in 2D with a level-symmetric quadrature set [15]. In this case $M=40$ and hence $\mathbf{T}^{ \pm}$are $80 \times 80$ matrices. Figs. 2-4 show the largest magnitude of eigenvalues

$$
\max _{k}\left|\omega_{k}\left(\tilde{\lambda}_{x}, \tilde{\lambda}_{y}\right)\right|
$$

over the Fourier plane $\left(\tilde{\lambda}_{x}, \tilde{\lambda}_{y}\right)$ computed for problems (i) without scattering $(c=0)$, (ii) with intermediate scattering ratio ( $c=0.5$ ), and (iii) with pure scattering $(c=1)$. The results are presented for a finite range of $\tilde{\lambda}_{x}$ and $\tilde{\lambda}_{y}$ because $\omega$ is a periodic function. We consider spatial grids with cells of characteristic optical thicknesses of $10^{-2}, 1$, and 10 . The results for other values of $c$ are similar. The analysis of the obtained data shows that the spectral radius equals 1 in all considered cases.

\section{Numerical results for finite-medium problems in $2 \mathrm{D}$}

The theoretical results of Fourier analysis of the ITMM for the DD scheme are obtained for infinite-medium problems. We now compare them with numerical results of $S_{8}$ finite-medium 2D problems for spatial domains $\mathcal{D}$ with $X=N_{x} \Delta x$ and $Y=N_{y} \Delta y$, homogeneous material and constant source $q=1$ everywhere in $\mathcal{D}$. Boundary conditions are vacuum $\left(\psi_{i n}=0\right)$. We use a random initial guess for face-average angular fluxes $\left(\psi_{m, k, j}^{\alpha-(0)}, \alpha=x, y\right)$. In all numerical tests, we apply the convergence criterion for cell-average scalar fluxes in the $\infty$-norm with a parameter that is equal to $10^{-12}$. To analyze the convergence of the method we calculate the difference in the face-average angular fluxes in two successive iterations measured in the $\infty$-norm 


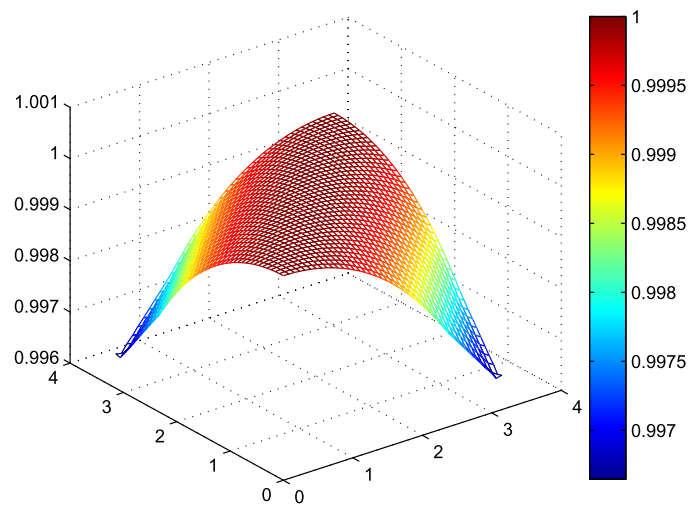

(a) $\sigma_{t} \Delta x=\sigma_{t} \Delta y=0.01$

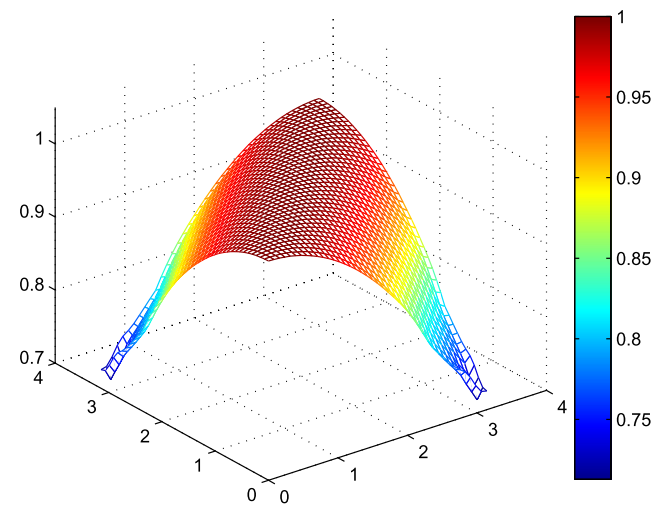

(b) $\sigma_{t} \Delta x=\sigma_{t} \Delta y=1$

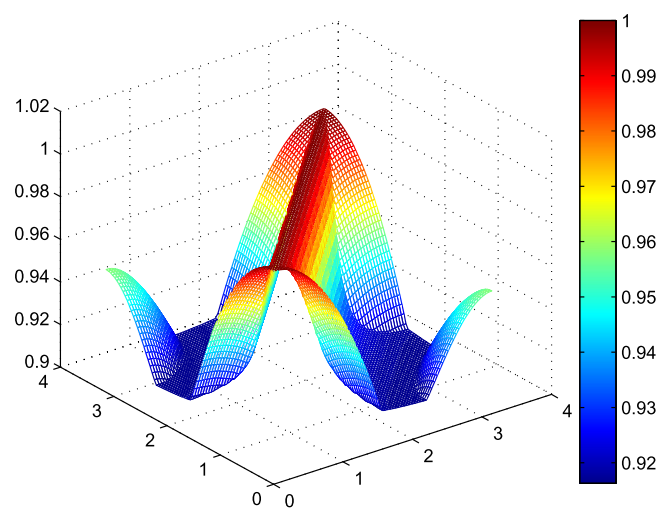

(c) $\sigma_{t} \Delta x=\sigma_{t} \Delta y=10$

Fig. 4. $\max _{k}\left|\omega_{k}\left(\tilde{\lambda}_{x}, \tilde{\lambda}_{y}\right)\right|$ for $S_{8}$ problems in $2 \mathrm{D}$ with $c=1$.

$$
\left\|\Delta \psi_{f}^{(s)}\right\|_{\infty}=\left\|\psi^{\alpha+(s)}-\psi^{\alpha+(s-1)}\right\|_{\infty},
$$

the difference in the cell-average scalar fluxes

$$
\left\|\Delta \phi_{c}^{(s)}\right\|_{\infty}=\left\|\phi^{(s)}-\phi^{(s-1)}\right\|_{\infty},
$$

and the rate of convergence inferred from the iterative errors in the angular and scalar fluxes:

$$
\rho_{\psi}^{(s)}=\frac{\left\|\Delta \psi_{f}^{(s)}\right\|_{\infty}}{\left\|\Delta \psi_{f}^{(s-1)}\right\|_{\infty}}, \quad \rho_{\phi}^{(s)}=\frac{\left\|\Delta \phi_{c}^{(s)}\right\|_{\infty}}{\left\|\Delta \phi_{c}^{(s-1)}\right\|_{\infty}} .
$$

First, we consider problems without scattering, i.e. the case of $c=0$. It takes a finite number of iterations to obtain the exact numerical transport solution of this class of problems. It depends on the number of spatial cells in the $x$ and $y$ directions and equals $N_{x}+N_{y}-1$. Fig. 5 shows the results in a square domain $N_{x}=N_{y}=256$ and $\Delta x=\Delta y=1$ with $\sigma_{t}=1$. The convergence histories of the angular fluxes $\left\|\Delta \psi_{f}^{(s)}\right\|_{\infty}$ are plotted in Fig. 5(a). Fig. $5(\mathrm{~b})$ shows $\left\|\Delta \phi_{c}^{(s)}\right\|_{\infty}$. Fig. 6 demonstrates the rate of convergence. The norm of error in the angular flux $\left\|\Delta \psi_{f}^{(s)}\right\|_{\infty}$ changes slowly and $\rho_{\psi}^{(s)}$ is very close to 1 . The exact discrete transport solution is obtained in 511 iterations and hence $\left\|\Delta \psi_{f}^{(s=512)}\right\|_{\infty}=\left\|\Delta \phi_{c}^{(s=512)}\right\|_{\infty}=0$. The errors in the scalar flux $\left\|\Delta \phi_{c}^{(s)}\right\|_{\infty}$ oscillate with larger amplitude than $\left\|\Delta \psi_{f}^{(s)}\right\|_{\infty}$. The amplitude decreases in the second half of the iterations.

We note that the exact angular flux is formed as a plane wave that moves from the incoming corner of the spatial domain corresponding to this ordinate. This wave propagates at the rate of one diagonal plane of cells per iteration. In this problem the wave reaches the domain diagonal in 256 iterations. This constitutes the first stage of iterations during which the wavefront expands by 2 cells with each iteration. In the second stage the wave of generation of the exact solution propagates from the diagonal to the opposite corners. The wavefront narrows, again by 2 cells per iteration. The obtained results demonstrate distinct behavior of iterative errors in these two stages. Another peculiar fact is that the exact scalar flux 


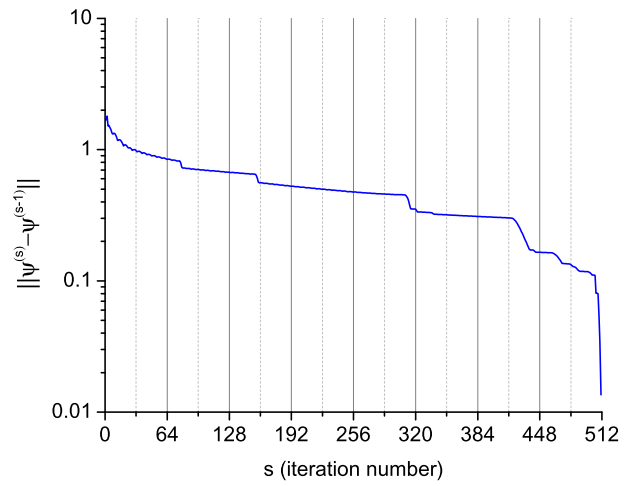

(a) $\left\|\Delta \psi_{f}^{(s)}\right\|_{\infty}$

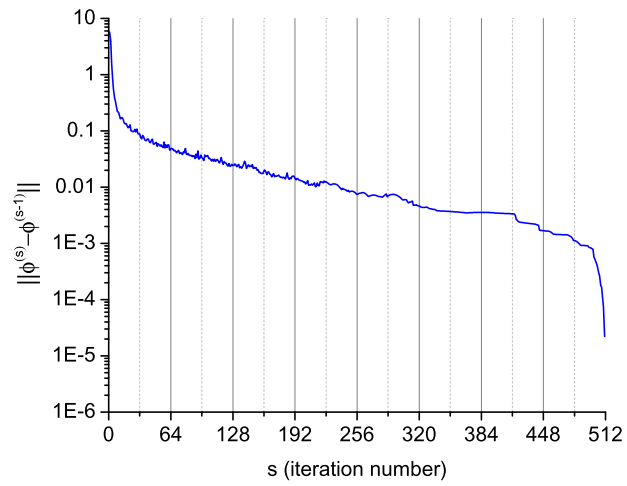

(b) $\left\|\Delta \phi_{c}^{(s)}\right\|_{\infty}$

Fig. 5. Convergence histories of ITMM for the model $S_{8}$ problem with $c=0, \sigma_{t} \Delta x=\sigma_{t} \Delta y=1$.

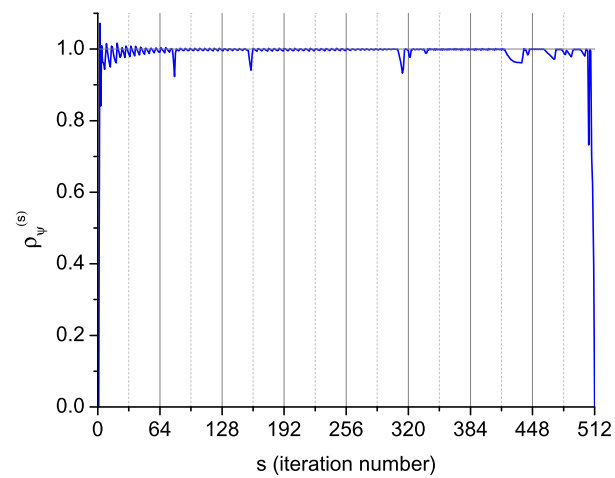

(a) $\rho_{\psi}^{(s)}$

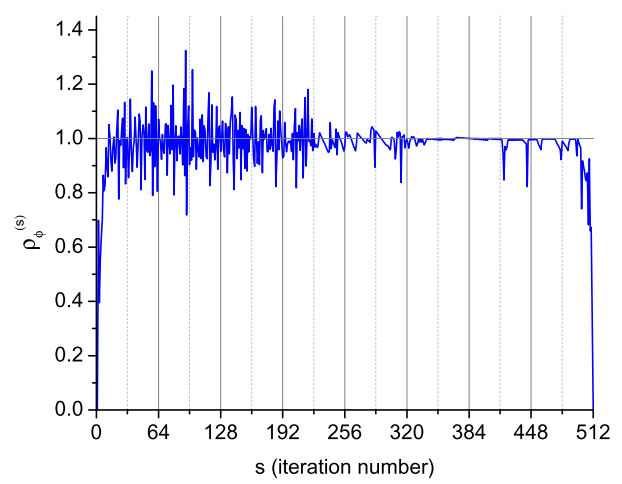

(b) $\rho_{\phi}^{(s)}$

Fig. 6. Rate of convergence of ITMM for the model $S_{8}$ problem with $c=0, \sigma_{t} \Delta x=\sigma_{t} \Delta y=1, N_{x}=N_{y}=256$.

is obtained in the center of the spatial domain at the end of the first stage. On the 256th iteration the exact face-average scalar fluxes are generated at the faces sharing the vertex located in the domain center. Then in the next iteration the first set of exact cell-average scalar fluxes is obtained in four cells around the center of the domain. This influences the behavior and magnitude of the iterative errors of the cell-average scalar flux during the rest of the iterations. In the second stage $(s \geq 257)$ the wave of generation of the exact scalar flux propagates from the center of the domain towards its boundary. The subdomain with the exact cell-average scalar flux grows each iteration by one neighboring cell around its boundary. This is only a partial explanation of ITMM iterative behavior in this class of problems. We note that some more effects of its iterative behavior remain to be interpreted.

Fig. 7 presents convergence histories for various values of the scattering ratio in the spatial domain with $N_{x}=N_{y}=256$ and $\sigma_{t} \Delta x=\sigma_{t} \Delta y=1$. The results for the case $c=0$ illustrate the phenomenon of generating uncollided flux by the ITMM in a finite number of iterations in problems with scattering $(c>0)$. This explains large and sharp reduction in iterative errors after the first $N_{x}+N_{y}-1$ iterations. Other instances of sharp decrease in iterative errors are related to recovering pieces of the transport solution associated with additional collisions. This happens every multiple of $N_{x}+N_{y}-1$ iterations. Between such instances the error changes with a regular rate. This observed pattern is due to the finite size of the spatial domain in contrast to the spectral analysis conducted for an infinite medium.

Fig. 8 shows the results in square domains $N_{x}=N_{y}$ and $\Delta x=\Delta y=1$ with $\sigma_{t}=1$ and an intermediate scattering ratio $c=0.5$. The number of cells is varied to increase the spatial domain's size. We notice that convergence of the ITMM iterations slows down with increasing size of the domain, i.e. as the finite domain tends towards the infinite domain employed in the Fourier analysis. Fig. 9 demonstrates the rate of convergence in the case of the largest spatial domain used in our numerical experiments $\left(N_{x}=N_{y}=256\right)$ computed using the $\infty$-norm of the iterative errors in the angular and scalar fluxes. These results show that the rate of convergence is very close to one. Thus, the rate of convergence tends to one as the problem domain size increases and leakage effects become weaker. Figs. 10 and 11 demonstrate performance in problems with small and large optical thicknesses of cells. The rate of convergence is again close to 1 . All obtained numerical results are consistent with theoretical results and confirm the prediction of the Fourier analysis. 


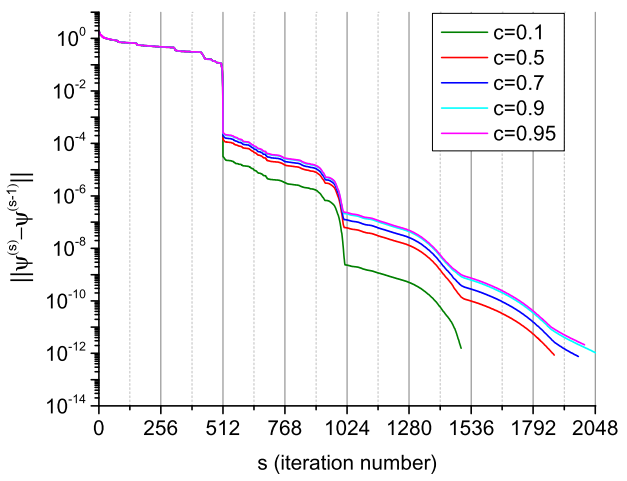

(a) $\left\|\Delta \psi_{f}^{(s)}\right\|_{\infty}$

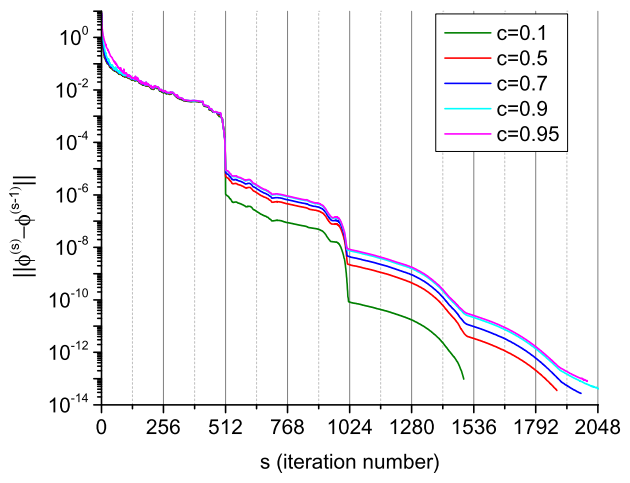

(b) $\left\|\Delta \phi_{c}^{(s)}\right\|_{\infty}$

Fig. 7. Convergence histories of ITMM for the model $S_{8}$ problem with various $c, \sigma_{t} \Delta x=\sigma_{t} \Delta y=1$ and $N_{x}=N_{y}=256$.

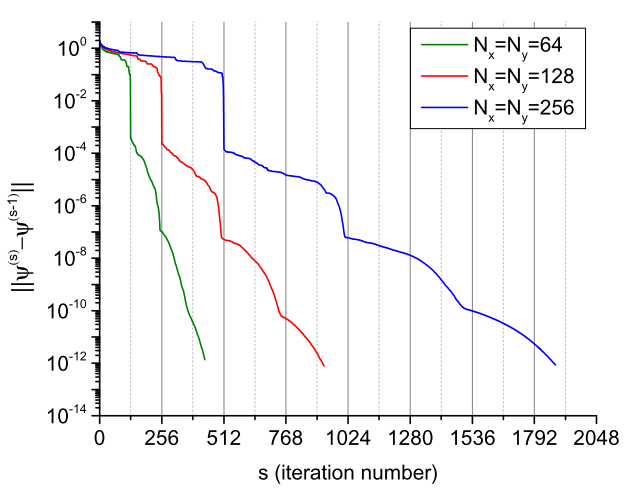

(a) $\left\|\Delta \psi_{f}^{(s)}\right\|_{\infty}$

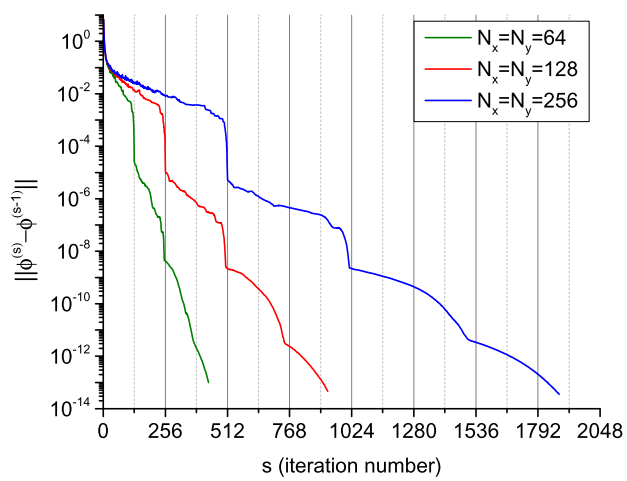

(b) $\left\|\Delta \phi_{c}^{(s)}\right\|_{\infty}$

Fig. 8. Convergence histories of ITMM for the model $S_{8}$ problem with $c=0.5, \sigma_{t} \Delta x=\sigma_{t} \Delta y=1$.

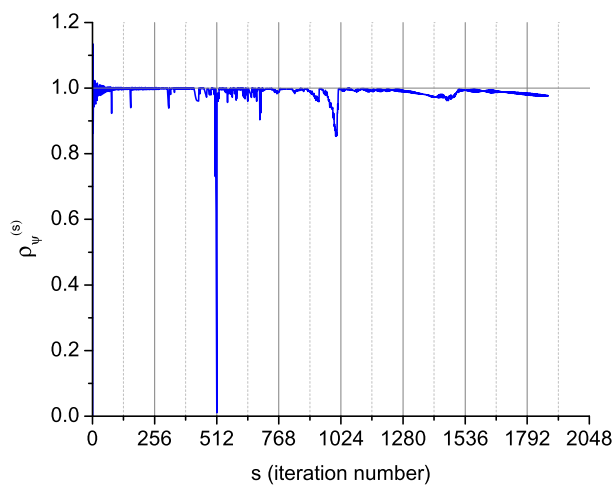

(a) $\rho_{\psi}^{(s)}$

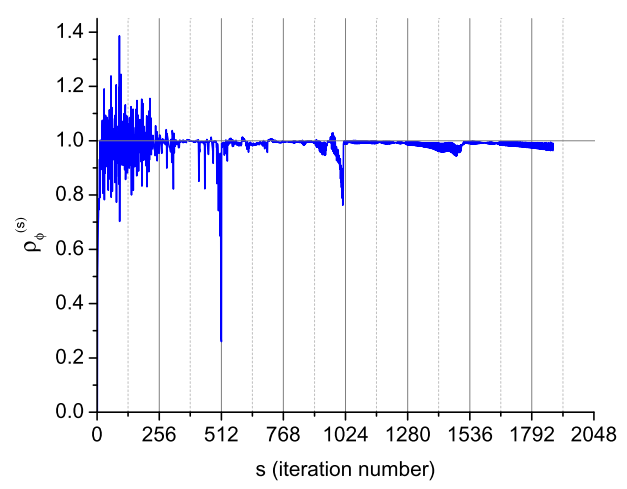

(b) $\rho_{\phi}^{(s)}$

Fig. 9. Rate of convergence of ITMM for the model $S_{8}$ problem with $c=0.5, \sigma_{t} \Delta x=\sigma_{t} \Delta y=1$, and $N_{x}=N_{y}=256$.

Convergence properties of the ITMM in the cases of small and high scattering ratios are similar. The results for $c=0.9$ and $c=1$ are presented in Figs. 12-15. In the last test, we see the effect of floating-point roundoff errors in calculations during the last thousand iterations when $\left\|\Delta \psi_{f}^{(s)}\right\|_{\infty}<10^{-12}$ and $\left\|\Delta \phi_{c}^{(s)}\right\|_{\infty}<10^{-12}$.

\section{Conclusion}

We have developed Fourier analysis of the integral transport matrix method based on a block Jacobi iterative strategy for the diamond-difference scheme for $S_{N}$ infinite-medium problems in the case of one cell per subdomain. This stability 


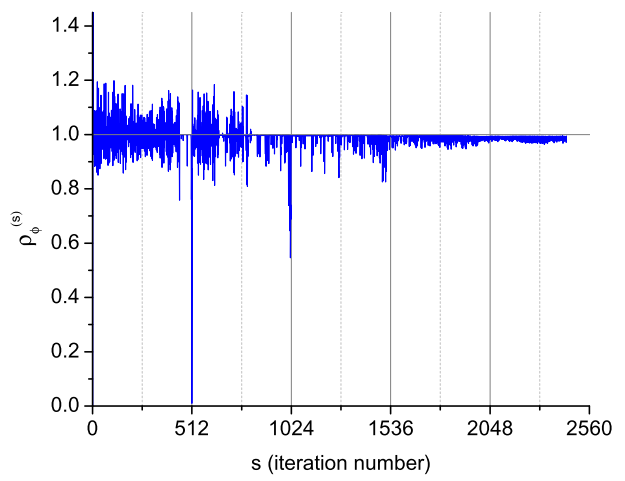

(a) $\rho_{\psi}^{(s)}$

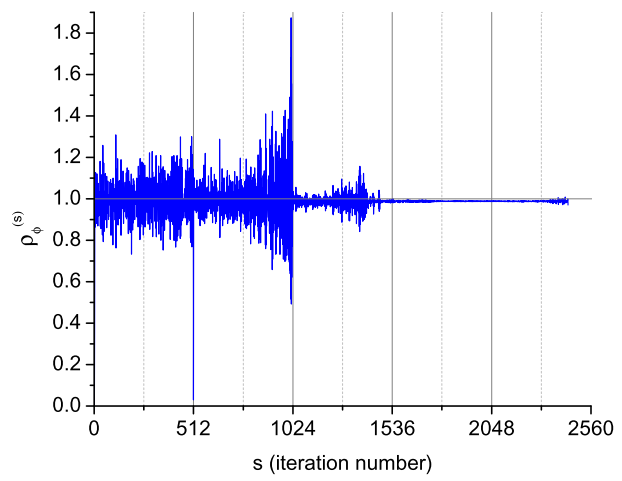

(b) $\rho_{\phi}^{(s)}$

Fig. 10. Rate of convergence of ITMM for the model $S_{8}$ problem with $c=0.5, \sigma_{t} \Delta x=\sigma_{t} \Delta y=0.01$, and $N_{x}=N_{y}=256$.

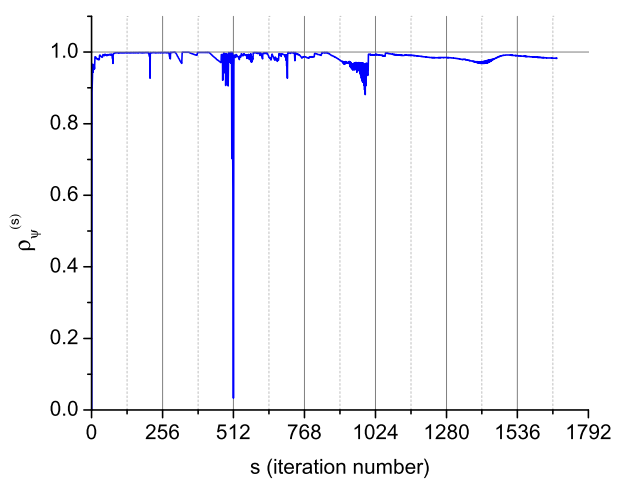

(a) $\rho_{\psi}^{(s)}$

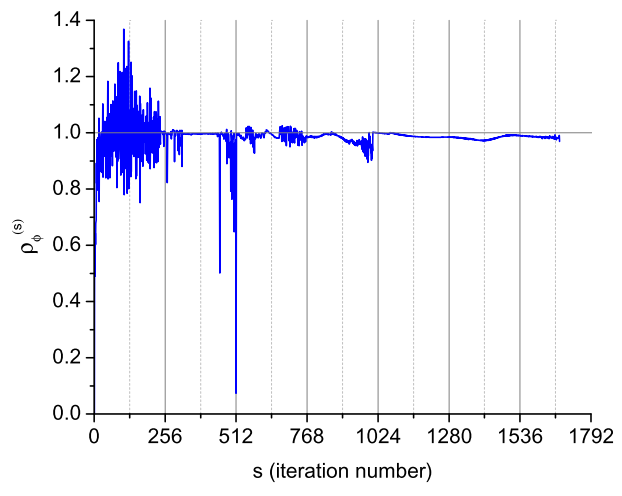

(b) $\rho_{\phi}^{(s)}$

Fig. 11. Rate of convergence of ITMM for the model $S_{8}$ problem with $c=0.5, \sigma_{t} \Delta x=\sigma_{t} \Delta y=10$, and $N_{x}=N_{y}=256$.

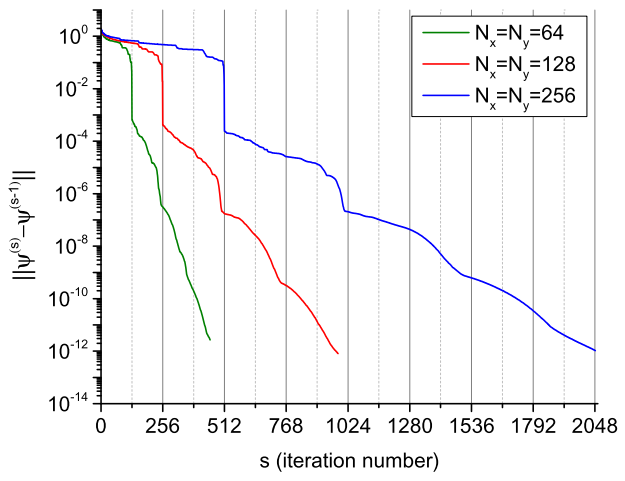

(a) $\left\|\Delta \psi_{f}^{(s)}\right\|_{\infty}$

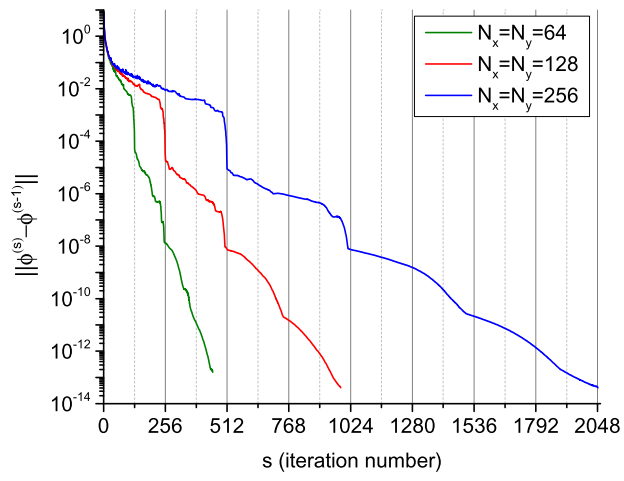

(b) $\left\|\Delta \phi_{c}^{(s)}\right\|_{\infty}$

Fig. 12. Convergence histories of ITMM for the model $S_{8}$ problem with $c=0.9, \sigma_{t} \Delta x=\sigma_{t} \Delta y=1$.

analysis enabled us to study convergence properties of the ITMM with the PBJ algorithm in this limiting case. It showed that in 2D geometry the spectral radius equals 1 without regard to values of the scattering ratio and optical thickness of the spatial cells. Numerical results confirmed these theoretical predictions. They demonstrated that the effectiveness of the ITMM for the DD scheme degrades with increasing size of the spatial domain in 2D Cartesian geometry. The results of tests with finite spatial domains revealed certain patterns in the behavior of iterative errors in these problems. This is an effect of the finite size of the spatial domain and related to generation of uncollided numerical solution and further recovering the transport solution associated with additional collisions. We also performed analysis of the ITMM with the PBJ method 


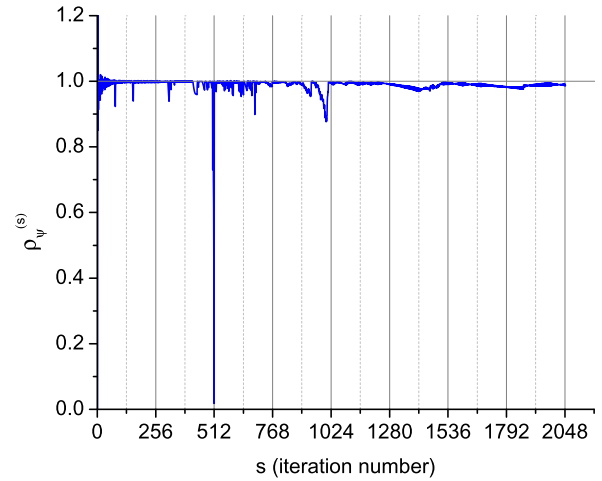

(a) $\rho_{\psi}^{(s)}$

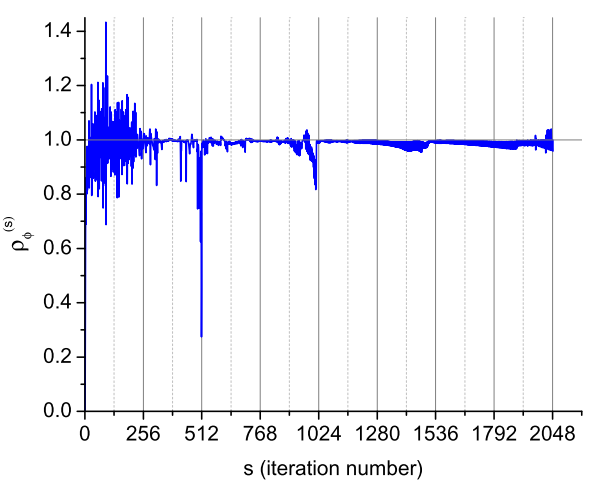

(b) $\rho_{\phi}^{(s)}$

Fig. 13. Rate of convergence of ITMM for the model $S_{8}$ problem with $c=0.9, \sigma_{t} \Delta x=\sigma_{t} \Delta y=1$, and $N_{x}=N_{y}=256$.

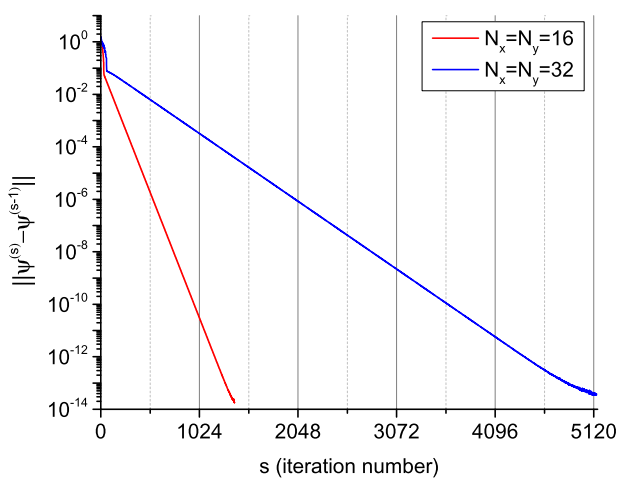

(a) $\left\|\Delta \psi_{f}^{(s)}\right\|_{\infty}$

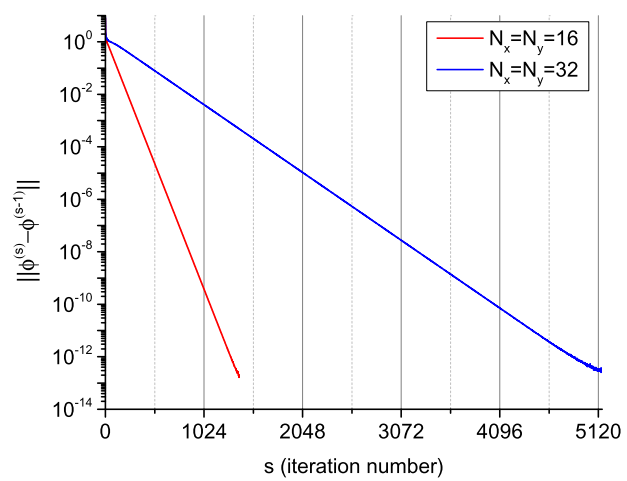

(b) $\left\|\Delta \phi_{c}^{(s)}\right\|_{\infty}$

Fig. 14. Convergence histories of ITMM for the model $S_{8}$ problem with $c=1, \sigma_{t} \Delta x=\sigma_{t} \Delta y=1$.

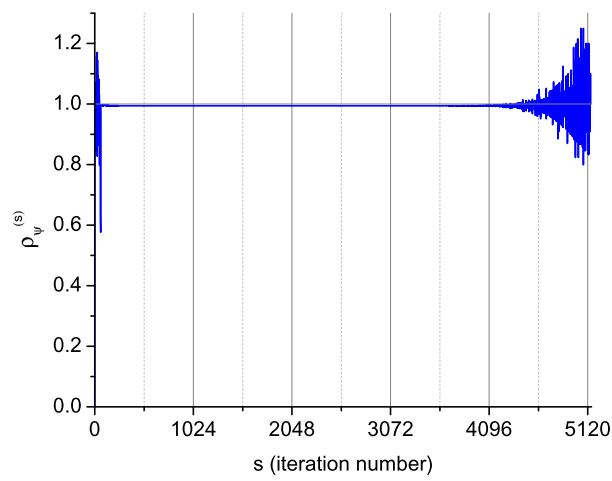

(a) $\rho_{\psi}^{(s)}$

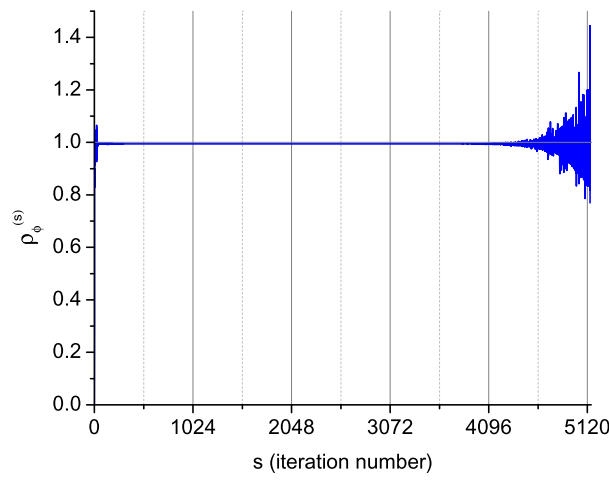

(b) $\rho_{\phi}^{(s)}$

Fig. 15. Rate of convergence of ITMM for the model $S_{8}$ problem with $c=1, \sigma_{t} \Delta x=\sigma_{t} \Delta y=1, N_{x}=N_{y}=32$.

for the DD scheme in 3D Cartesian geometry. The obtained results are very similar to those for 2D presented in this paper. The spectral radius in the limiting case of one cell per subdomain equals one.

The Fourier analysis developed in this paper can be extended to other transport discretization methods, for instance, the step and AHOT-NO methods that are different from the DD scheme by weights in auxiliary equations. Further work will address extra features of the iteration method, more general cases and different modifications of iterative algorithms for parallel computations. Such studies will help to find approaches for improving stability properties of the ITMM for the DD and other discretization schemes. 
Preliminary spectral analysis for the ITMM iterations using the AHOT-N0 [19] spatial discretization of the $S_{N}$ equations suggests that the spectral radius is generally smaller than one. As the cells get thinner the spectral radius approaches unity, an expected result since the AHOT-NO equations tend to the DD equations in this limit. Additionally, for the same cell size, the spectral radius appears to increase with increasing scattering ratio. These results will be verified with numerical experiments and reported separately.

\section{Acknowledgements}

This research was supported in part by the DOE Office of Nuclear Energy's Nuclear Energy University Programs under contract AC07-05ID14517. One of us (YYA) acknowledges useful discussions with Dr. Joe Zerr of LANL.

\section{References}

[1] J.J. Duderstadt, W.R. Martin, Transport Theory, John Wiley \& Sons, 1979.

[2] D. Mihalas, B. Weibel-Mihalas, Foundations of Radiation Hydrodynamics, Oxford University Press, 1984

[3] B.R. Wienke, R.E. Hiromoto, Parallel $S_{N}$ transport algorithms, Transp. Theory Stat. Phys. 15 (1986) 49.

[4] M. Yavuz, E.W. Larsen, Spatial domain decomposition for neutron transport problems, Transp. Theory Stat. Phys. 18 (1989) 205.

[5] Y.Y. Azmy, Communication strategies for angular domain decomposition of transport calculations on message passing multiprocessors, in: Proc. of Int. Conf. on Math. Methods and Supercomputing for Nucl. Appl., vol. 1, Saratoga Springs, NY, 1997, p. 404.

[6] R.S. Baker, R.E. Alcouffe, Parallel 3-D $S_{N}$ performance for DANTSYS/MPI on the Cray T3D, in: Proc. of Int. Conf. on Math. Methods and Supercomputing for Nucl. Appl., vol. 1, Saratoga Springs, NY, 1997, p. 377.

[7] R.S. Baker, K.R. Koch, An $S_{N}$ algorithm for the massively parallel CM-200 computer, Nucl. Sci. Eng. 128 (1998) 312.

[8] M. Rosa, J.S. Warsa, J.H. Chang, Fourier analysis of inexact parallel block-Jacobi splitting with transport synthetic acceleration, Nucl. Sci. Eng. 164 (2010) 248-263.

[9] M.P. Adams, M.L. Adams, W.D. Hawkins, T. Smith, L. Rauchwerger, N.M. Amato, T.S. Bailey, R.D. Falgout, Provably optimal parallel transport sweeps on regular grids, in: Proc. of M\&C 2013, Int. Conf. on Math. and Comp. Methods Applied to Nucl. Sci. \& Eng., Sun Valley, Idaho, USA, 2013, pp. $2535-2553$.

[10] G.G. Davidson, T.M. Evans, J. Jarrell, S.P. Hamilton, T.M. Pandya, R.N. Slaybaugh, Massively parallel, three-dimensional transport solutions for the keigenvalue problem, Nucl. Sci. Eng. 177 (2014) 111-125.

[11] Y.Y. Azmy, A new algorithm for generating highly accurate benchmark solutions to transport test problems, in: Proc. of the XI ENFIR / IV ENAN Joint Nuclear Conf., Pocos de Caldas Springs, MG, Brazil, August 18-22, 1997.

[12] M. Rosa, Y.Y. Azmy, J.E. Morel, Properties of the $S_{N}$-equivalent integral transport operator in slab geometry and the iterative acceleration of neutral particle transport methods, Nucl. Sci. Eng. 162 (2009) 234-252.

[13] R.J. Zerr, Y.Y. Azmy, A parallel algorithm for solving the multidimensional within-group discrete ordinates equations with spatial domain decomposition, in: Proc. of PHYSOR 2010, Advances in Reactor Phys. to Power the Nucl. Renaissance, Pittsburgh, PA, USA, 2010.

[14] R.J. Zerr, Y. Azmy Y, Improved parallel solution techniques for the integral transport matrix method, in: Proc. of M\&C 2011, Int. Conf. on Math. and Comp. Methods Applied to Nucl. Sci. \& Eng., Rio de Janeiro, RJ, Brazil, 2011.

[15] E.E. Lewis, W.F. Miller Jr., Computational Methods of Neutron Transport, American Nuclear Society, La Grange Park, IL, USA, 1993.

[16] E.E. Lewis, I. Dilber, Finite element, nodal and response methods: a variational synthesis for neutron transport, Prog. Nucl. Energy 18 (1986) 63-74.

[17] E.W. Larsen, Diffusion-synthetic acceleration methods for discrete-ordinates problems, Transp. Theory Stat. Phys. 13 (1984) $107-126$.

[18] R. Sanchez, On the spectral analysis of iterative solutions of the discretized one-group transport equation, Ann. Nucl. Energy 31 (2004) 2059-2075.

[19] Y.Y. Azmy, General order nodal transport methods and application to parallel computing, Transp. Theory Stat. Phys. 22 (1993) 359. 